\title{
Prompt and Long-Lived Anti-Kasha Emission from Organic Dyes
}

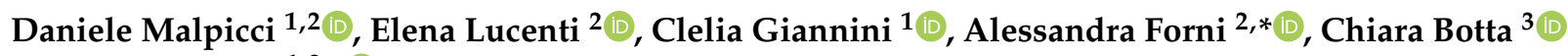 \\ and Elena Cariati $1,2, *$ (i)
}

1 Dipartimento di Chimica, Università degli Studi di Milano and INSTM RU, Via Golgi 19, 20133 Milano, Italy; daniele.malpicci@unimi.it (D.M.); clelia.giannini@unimi.it (C.G.)

2 Institute of Chemical Sciences and Technologies "Giulio Natta" (SCITEC) of CNR and INSTM RU, Via Golgi 19, 20133 Milano, Italy; elena.lucenti@scitec.cnr.it

3 Institute of Chemical Sciences and Technologies "Giulio Natta" (SCITEC) of CNR and INSTM RU, Via Corti 12, 20133 Milano, Italy; chiara.botta@scitec.cnr.it

* Correspondence: alessandra.forni@scitec.cnr.it (A.F.); elena.cariati@unimi.it (E.C.)

Citation: Malpicci, D.; Lucenti, E.; Giannini, C.; Forni, A.; Botta, C.;

Cariati, E. Prompt and Long-Lived Anti-Kasha Emission from Organic Dyes. Molecules 2021, 26, 6999. https://doi.org/10.3390/ molecules26226999

Academic Editors: Rui Fausto and Scott Reed

Received: 20 October 2021

Accepted: 15 November 2021

Published: 19 November 2021

Publisher's Note: MDPI stays neutral with regard to jurisdictional claims in published maps and institutional affiliations.

Copyright: (C) 2021 by the authors Licensee MDPI, Basel, Switzerland. This article is an open access article distributed under the terms and conditions of the Creative Commons Attribution (CC BY) license (https:/ / creativecommons.org/licenses/by/ $4.0 /)$.

\begin{abstract}
Anti-Kasha behavior has been the subject of intense debate in the last few years, as demonstrated by the high number of papers appearing in the literature on this topic, dealing with both mechanistic and applicative aspects of this phenomenon. Examples of anomalous emitters reported in the last 10 years are collected in the present review, which is focused on strictly anti-Kasha organic molecules displaying radiative deactivation from $S_{n}$ and/or $T_{n}$, with $n$ greater than 1 .
\end{abstract}

Keywords: anti-Kasha fluorescence; anti-Kasha phosphorescence; organic dyes

\section{Introduction}

In its original formulation, the Kasha rule states that for complex organic molecules, "regardless of which electronic state of a given multiplicity is excited [ ... ], the emitting electronic level of a given multiplicity is the lowest excited level of that multiplicity" [1] This rule originates from the widespread feature that the $E_{1}-E_{0}$ energy gap is much larger than the $E_{n}-E_{n-1}(n>1)$ ones and the energy gap law. According to this law, the smaller the energy gap between two electronic states is, the more probable is the nonradiative deactivation (by IC and ISC) between them. In the condensed phase, exceptions to Kasha's rule are possible under various circumstances. In particular, two prototype mechanisms are responsible for fluorescence from $S_{2}$ : (i) when the energy gap between $S_{1}$ and $S_{2}$ is large and the oscillator strength $(f)$ of the $S_{0}-S_{2}$ transition is large, fluorescence from $S_{2}$ becomes competitive, a mechanism observed for example for azulene and its derivatives [2-5] and thioketones [6-8], the difference between the two families being the $\left(\pi, \pi^{*}\right)$ character of $S_{1}$ for the former and $\left(n, \pi^{*}\right)$ for the latter; (ii) when the $S_{2}-S_{1}$ energy gap and $f$ of the $\mathrm{S}_{0}-\mathrm{S}_{1}$ transition are very small but $f$ of the $\mathrm{S}_{0}-\mathrm{S}_{2}$ transition is large, fluorescence from $S_{2}$ is observed due to thermal population from $S_{1}$, a mechanism observed for example for ovalene [9]. Moreover, fluorescence from higher $S_{n}(n>2)$ can be observed when energy separation between the levels is favorable (necessary condition) and the $S_{n}-S_{1}$ internal conversion is prohibited based on symmetry grounds (sufficient condition) [10]. AntiKasha phosphorescence from high-energy triplet levels is of course less observed. It may be favored by either an easy ISC (by both El-Sayed and energy gap considerations) to $T_{2}$ level of proper symmetry, different from the $\mathrm{T}_{1}$ one so as to give low $\mathrm{T}_{2}-\mathrm{T}_{1} \mathrm{IC}$ efficiency, or $\mathrm{T}_{2}$ thermal population from $\mathrm{T}_{1}$.

Currently, it is proven that the Kasha rule applies to most of the emissive organic molecules, and despite the increased instrumental sensitivity with respect to that available when Kasha made his observation, it is difficult to recognize exceptions. However, "anti-Kasha" emissions represent interesting cases for both fundamental knowledge and 
application aspects. In fact, anti-Kasha emitters do not dissipate through IC the excess of electronic energy, which can therefore be exploited in practical applications, such as improved luminescent quantum efficiency, tunable emission colors and lifetimes. The interest in this subject is demonstrated by the increasing number of works appearing in the literature in the last few years. In this regard, in 2012, Itoh reviewed works produced until 2011 on traditional anti-Kasha emitters such as azulene and its derivatives, aromatic acenes, polyenes, thioketones, metalloporphyrins, aromatic carbonyl compounds, quinones and halogenated aromatic compounds [9]. The reported examples comprise emission from higher singlet and triplet levels.

Anomalous emission includes a large number of photophysical phenomena, among which anti-Kasha behavior represents a very limited subset that manifests either through single or multiple emissions [11]. The first case may be particularly difficult to discern because low-lying dark excited levels are silent to many experimental investigations. Importantly, when more than one band is displayed by a single molecule due to structural rearrangement and/or chemical transformation, such as "excited state conjugation enhancement" (ESCE), "twisted intramolecular charge transfer" (TICT), "excited state intramolecular proton transfer" (ESIPT) and "optically triggered counterion migration" (OTCM) [11], dual or multiple emissive behavior is to be used instead of anti-Kasha behavior. Actually, a few examples of ESCE, TICT, ESIPT and OTCM associated with anti-Kasha behavior exist [12] since the reorganization occurs starting from a higher excited state in the molecule. However, such examples will not be covered in the present review. Even those cases where various emissions are due to the molecule and its aggregates in the same phase (solution, amorphous or crystalline solids) have to be described as Kasha emitters. Of course, trivial mistakes such as the presence of traces of impurities which can be responsible for the appearance of multiple emission bands should be carefully checked before claiming an anti-Kasha behavior $[13,14]$.

Here we collect works reporting "rigorous" anti-Kasha emitters that have appeared in the literature since 2011, starting from the seminal analysis from Itoh [9]. By "rigorous", we mean systems that radiatively deactivate from $S_{n}$ or $T_{n}(n>1)$. In fact, in the literature, some works report as anti-Kasha a behavior that instead could be better described as anti-Vavilov $[14,15]$. According to the Vavilov rule, the intensity of the dye emissions is independent of excitation wavelength.

Another important caveat should be presented. Since anti-Kasha behavior is hardly recognizable through an exclusively experimental approach, in particular when states below the emissive $S_{n}$ or $T_{n}(n>1)$ ones are silent, quantum-chemical computational studies acquire a key role in establishing the presence and the origin of anomalous emission. Owing to the high computational costs associated with accurate $a b$ initio techniques (e.g., CASPT2 and MRCI), most theoretical investigations aimed at predicting electronic spectra of complex molecules are performed within the TDDFT approach. The performance of this method was always deemed highly reliable for predicting non-charge-transfer excitations, while it is well known that exchange-correlation functionals generally used for ground-state calculations, such as those based on the generalized gradient approximation (GGA) or the hybrid GGA functionals (typically the popular B3LYP one) do not allow for sufficient accuracy to study CT excited states [16-21]. Very few exceptions (for example, the PBE0 functional) have been recognized [22]. Recently, the development of the so-called range-separated hybrid functionals (including CAM-B3LYP, $\omega B$ 97X and $\omega B$ 97XD), where the exchange term is split into long- and short-range [23-27] and the range-separation parameter can be suitably determined by a self-consistent procedure [28], has allowed for easy resolution of the CT problem for many systems of practical interest [29,30]. Additional functionals have been developed since, such as the hybrid meta-GGA ones (including Mo6-2X) whose performance in electronic spectra calculations has been assessed [31]. It is therefore to be noted that the cases of anti-Kasha behavior involving CT excited states collected in the present review, especially those where TDDFT calculations have been performed using inadequate functionals, could be confuted by more reliable approaches. 
This manuscript is organized by different families of molecules, including azulenes, cyclic triimidazoles, 1,2-diphenylphenanthroimidazole derivatives, cyanines, thiophenes and carbazoles.

\section{Azulenes}

Azulene, 1 (Scheme 1), can be regarded as the forefather of organic molecules displaying anti-Kasha behavior, namely a fluorescent emission from the $S_{2}$ excited state $[9,32]$.

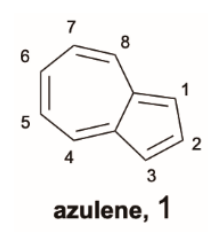

Scheme 1. Chemical structure of compound 1.

This feature derives from the unique molecular structure of azulene, different from other traditional aromatic compounds and characterized by an electron-rich five-membered ring fused with an electron-poor seven-membered ring with HOMO density localized mainly on the odd positions and LUMO density located on the even positions [33] (Figure 1). This leads to an unusually low-lying first electronic excited state $\left(S_{1}\right)$, favoring a large $\mathrm{S}_{2}-\mathrm{S}_{1}$ energy gap $(>1.2 \mathrm{eV})$ and, according to the energy-gap law, resulting in fluorescence from $S_{2}$ rather than IC to $S_{1}$. Similar emissive behavior is found for variously substituted azulenes, polyazulenes and pseudoazulenes (having a $-\mathrm{CH}=\mathrm{CH}-$ group in the heptagonal ring of azulene replaced by a heteroatom). It has been shown that HOMO and LUMO energy levels can be modified by proper choice of electron-donor or electronwithdrawing functional groups. In particular, the insertion of electron-donor substituents at the 1, 3, 5 or 7 ring positions usually increases the $S_{2}-S_{1}$ gap while electron-withdrawing substituents have the opposite effect. A reverse behavior is found for substitutions at the 2, 4,6 and 8 positions, resulting in an $S_{2}-S_{1}$ energy gap increased by electron-withdrawing groups and lowered by electron-donor substituents. In some cases, for derivatives characterized by a relatively small $S_{2}-S_{1}$ energy gap, IC to $S_{1}$ becomes competitive with fluorescence from $S_{2}$, and fluorescence from both excited states is observed [9,32]. Azulene and a series of its derivatives have been the subject of accurate theoretical benchmark studies to validate state-of-the-art computational protocols predicting anti-Kasha photoluminescence [34,35].
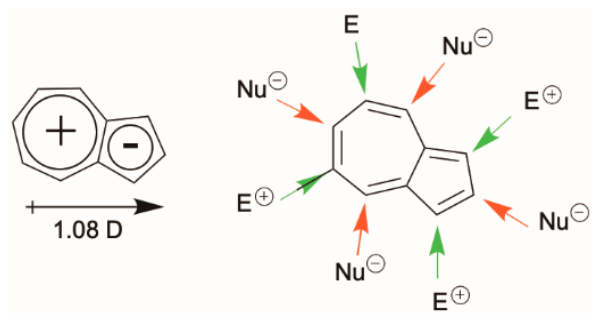

Figure 1. Polarized resonance structure of azulene with the dipole moment (left) and reactivity of each position of azulene (right). Adapted with permission from [33], Copyright 2021, American Chemical Society.

Due to these intriguing photophysical properties, there is a growing interest in the development of azulene-based materials for optoelectronic applications, even though the preparation of new derivatives is hampered by multistep and low-yield synthetic procedures $[32,33,36]$.

In this regard, Zhou et al. [37] reported on two azulene derivatives, 2 and 3 (Scheme 2), substituted at the 3 position with cyanostyryl moieties that, under UV or visible irradiation in acidic conditions, photoisomerize from Z- to E-form (Figure 2). The process is accompanied by a strong modification of the photoluminescent features. In particular, nonradiative 
decays are inhibited by sterical restrictions going from the not emissive Z-isomer to the E-isomer. Moreover, the E-form displays dual luminescence with a major emission band at around $480 \mathrm{~nm}$ (assigned to an $\mathrm{S}_{2}-\mathrm{S}_{0}$ transition) and an NIR emission at around $800 \mathrm{~nm}$ (attributed to a radiative decay from $S_{1}$, Figure 3). The conversion between the two isomers is reversible, and the E-isomer can be converted into the Z-isomer by simply heating the sample at a temperature above $65^{\circ} \mathrm{C}$.

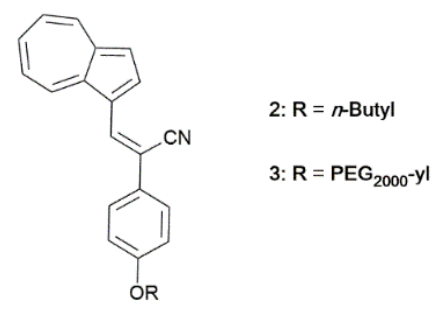

Scheme 2. Chemical structure of compounds 2 and 3.

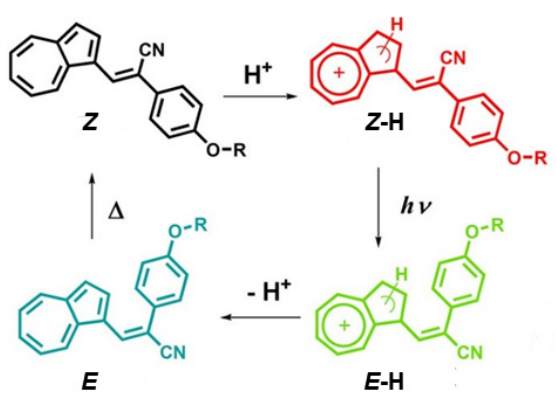

Figure 2. (Top) Chemical structures of the cyanostyryl-modified azulenyl compounds 2 and 3. (Bottom) Protonation-assisted photoisomerization process. Adapted with permission from [37].
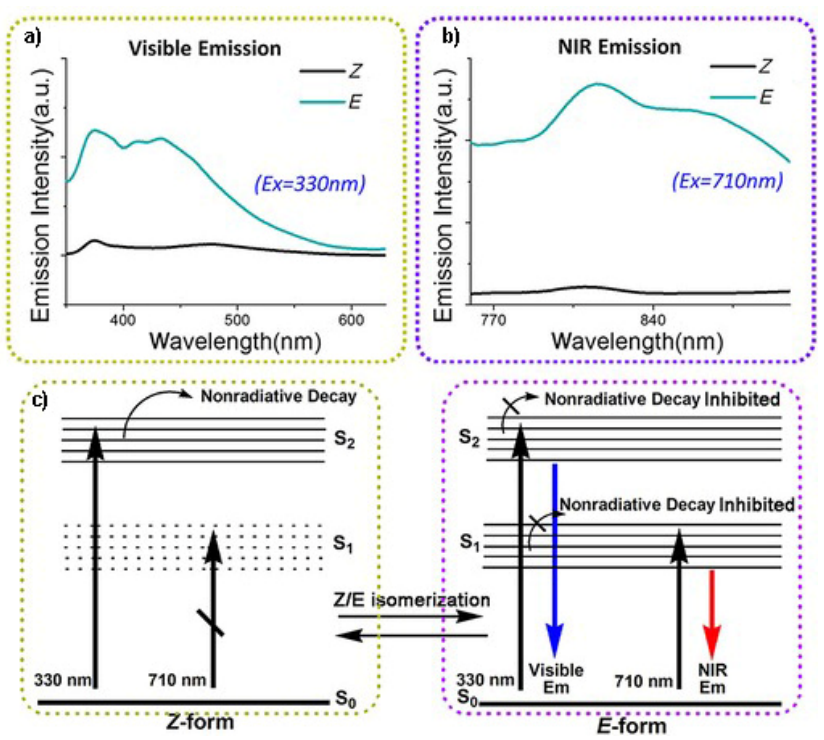

Figure 3. Emission spectra of 3 in Z- and E-form in water $\left(5 \times 10^{-5} \mathrm{~mol} \mathrm{~L}^{-1}\right)$ at RT. (a) $\lambda_{\mathrm{ex}}=330 \mathrm{~nm}$, scanning range $=350-900 \mathrm{~nm} ;(\mathbf{b}) \lambda_{\mathrm{ex}}=710 \mathrm{~nm}$, scanning range $=730-900 \mathrm{~nm}$. (c) Proposed mechanism for the tuning of the dual pathway luminescence in Z- and E-form. Adapted with permission from [37].

The same research group investigated the photophysics of compound 4 (Scheme 3) in which dual Vis and NIR emissive cyanostyryl-modified azulene moieties are inserted onto a hexathiobenzene core [38]. With respect to its cyanostyryl-azulene precursor, 4 displays an increased anti-Kasha emission at $480 \mathrm{~nm}$ due to a FRET process from the hexathiobenzene 
to the azulene. The emission intensity of 4 can be further enhanced in tetrahydrofuran (THF) $/ \mathrm{H}_{2} \mathrm{O}$ solvent mixtures by an AIE effect, thus leading to a total 15-fold amplification of the QY of this material compared to its precursors. In addition, 4 is a rare example of multiwavelength anti-Kasha emissions since it displays, in addition to the visible $S_{2}-S_{0}$ fluorescence at $480 \mathrm{~nm}$, a UV emission at $360 \mathrm{~nm}$, generated by radiative decay from the higher $S_{3}$ excited state. Interestingly, these emissive features are retained in PMMA films, opening the way to possible applications of solid-state anti-Kasha materials.

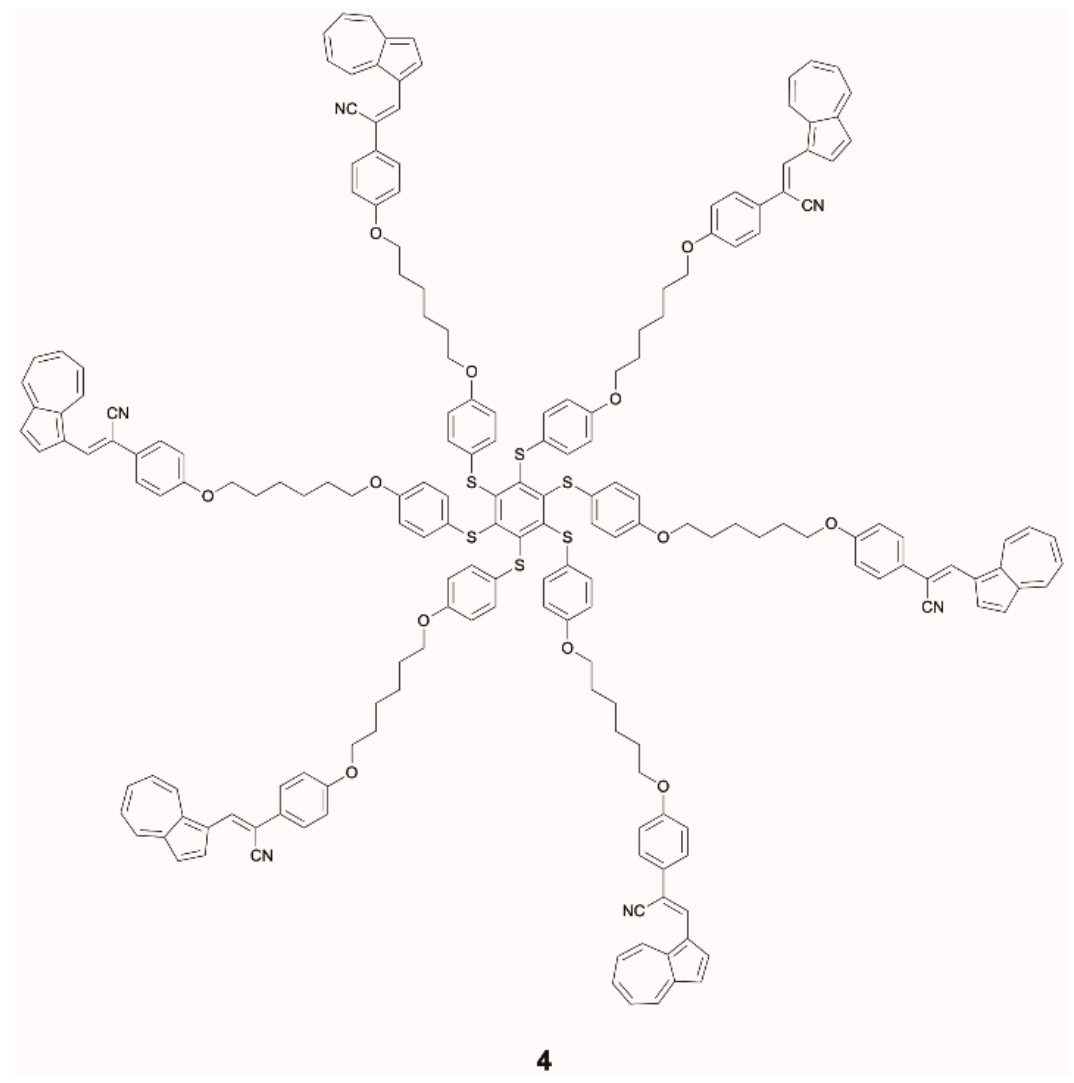

Scheme 3. Chemical structure of compound 4 .

In an extension of their research, Zhou et al. [39] reported on a series of mono-, diand triformyl-substituted azulenes, among which the triformyl derivative 5 (Scheme 4) shows a remarkable dual fluorescence (photoluminescence QY, $\Phi$, up to $10 \%$ ) in DMF solution. On the basis of experimental and theoretical investigations, the latter based on TDDFT calculations at the B3LYP/6-31G(d) level of theory, the photoluminescent behavior of 5 was attributed to concomitant radiative deactivations from the upper $S_{3}$ and $S_{2}$ energy levels with $\left(\pi, \pi^{*}\right)$ and $\left(n, \pi^{*}\right)$ character, respectively, and having an energy gap of $0.35 \mathrm{eV}$ which guarantees a slow IC. In the presence of water, intermolecular H-bonds between the aldehydic groups and water result in the red shift of the emission and in the quenching of the $S_{3}$ deactivation path due to the stabilization of $S_{4}$ and $S_{5}\left(n, \pi^{*}\right)$ levels (see Figure $4 \mathrm{~b}$ ). Tunable emission color, from blue to cyan and green, by H-bonding control was demonstrated both in solution and in the solid state by using hydrophilic polymers such as polyvinyl alcohol (PVA) and polyacrylic acid sodium salt (PAAS) as matrices to ensure a sufficient moisture sensitivity and the realization of a visual sensor for moisture (Figure 4c). 


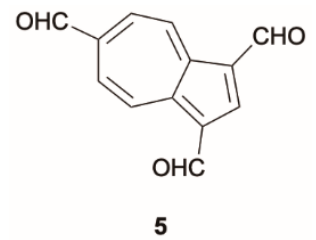

Scheme 4. Chemical structure of compound 5.

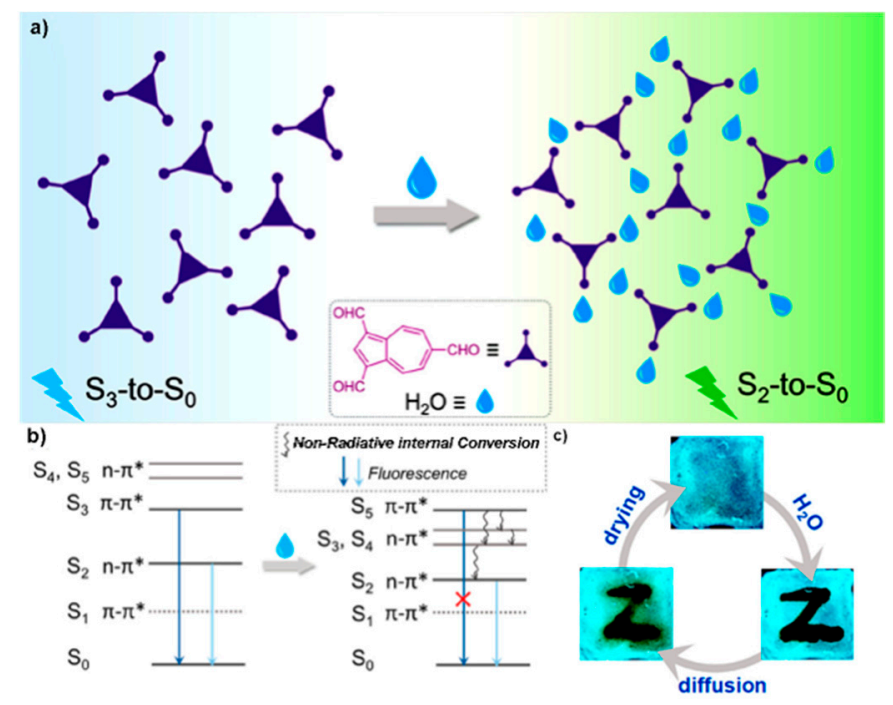

Figure 4. (a) Proposed process for the formation of intermolecular H-bonding of compound 5 upon addition of a small amount of $\mathrm{H}_{2} \mathrm{O}$. (b) Proposed mechanism accompanied by an anti-Kasha luminescent conversion for the tuning of the 5 luminescence in $\mathrm{DMF} / \mathrm{H}_{2} \mathrm{O}$ mixtures. (c) The writing of " $\mathrm{Z}$ " character with $\mathrm{H}_{2} \mathrm{O}$ onto the 5-doped PVA/PAAS film on a quartz substrate and the process of the three-state recyclable (photographs were taken under $365 \mathrm{~nm}$ UV light). Adapted with permission from [39], Copyright 2018, American Chemical Society.

Zhang et al. [40] reported on the synthesis of dicyclohepta[ij-kl,uvwx]rubicene, 6 (Scheme 5), a polycyclic aromatic hydrocarbon containing two coplanar pentagons and heptagons constituting two formal azulene moieties. The presence of these conjugated rings ensures thermal and air stability together with planarity, as confirmed by crystal structure analysis. A combined photophysical and theoretical investigation revealed the presence in THF solution of two emission bands at 670 and $400 \mathrm{~nm}$ which were assigned, according to CASPT2 $/ 6-31 G(d) /(12,12)$ calculations based on CASSCF orbitals, to $S_{1}-S_{0}$ and $S_{2}-S_{0}$ radiative decays, respectively. The anti-Kasha emission was established to originate mainly from a contribution of the two formal azulene units present in the molecular structure.

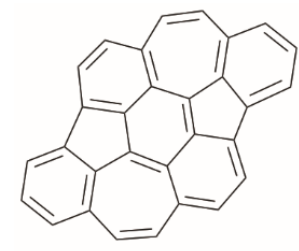

6

Scheme 5. Chemical structure of compound 6.

Based on the observation that protonation of azulene can result in a tropylium form with intensified fluorescence due to the suppression of anti-Kasha emission in favor of the $S_{1}$ one, Amir et al. [41] studied 5,5'-(azulene-4,7-diyl)bis(3-dodecylthiophene), 7 , and 2,2'-(6-dodecylazulene-4,7-diyl)dithiophene, 8 (Scheme 6). The two compounds can, in fact, 
undergo a reversible protonation/deprotonation process by treatment with trifluoroacetic acid (TFA) and triethylamine ( $\left.\mathrm{NEt}_{3}\right)$. The acidochromic switch can be easily followed by fluorescent spectroscopy (see Figure 5) revealing a red shift and a remarkable increase in emission intensity upon protonation and the quenching of the signal by deprotonation with the base.

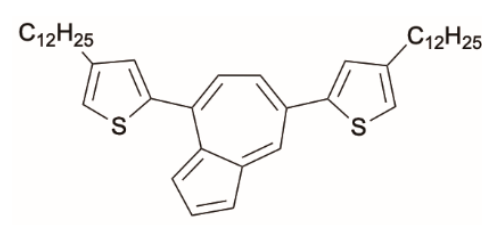

7

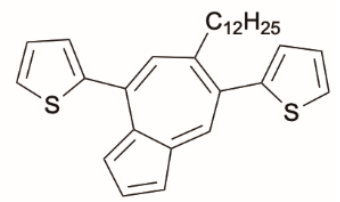

8

Scheme 6. Chemical structure of compounds 7 and 8.

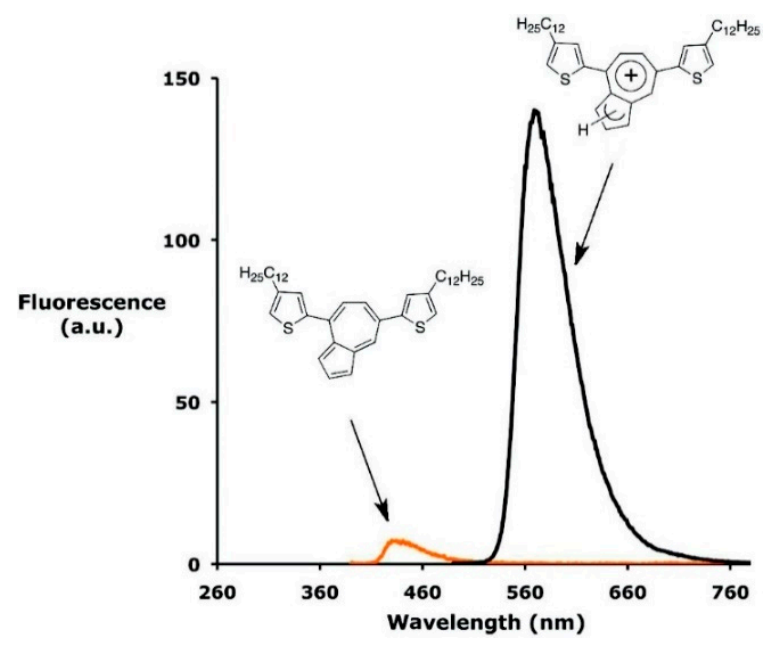

Figure 5. Fluorescence measurements in dichloromethane for neutral oligomer 7 and after protonation to give $\mathbf{7} \mathbf{H}^{+}$. Reprinted with permission from [41], Copyright 2011, American Chemical Society.

A similar behavior was reported by Koch et al. [42] for a series of 2-alkynyl azulenes, 9-20 (Scheme 7), characterized by a weak fluorescence that can be enhanced and red-shifted by treatment with acids. 


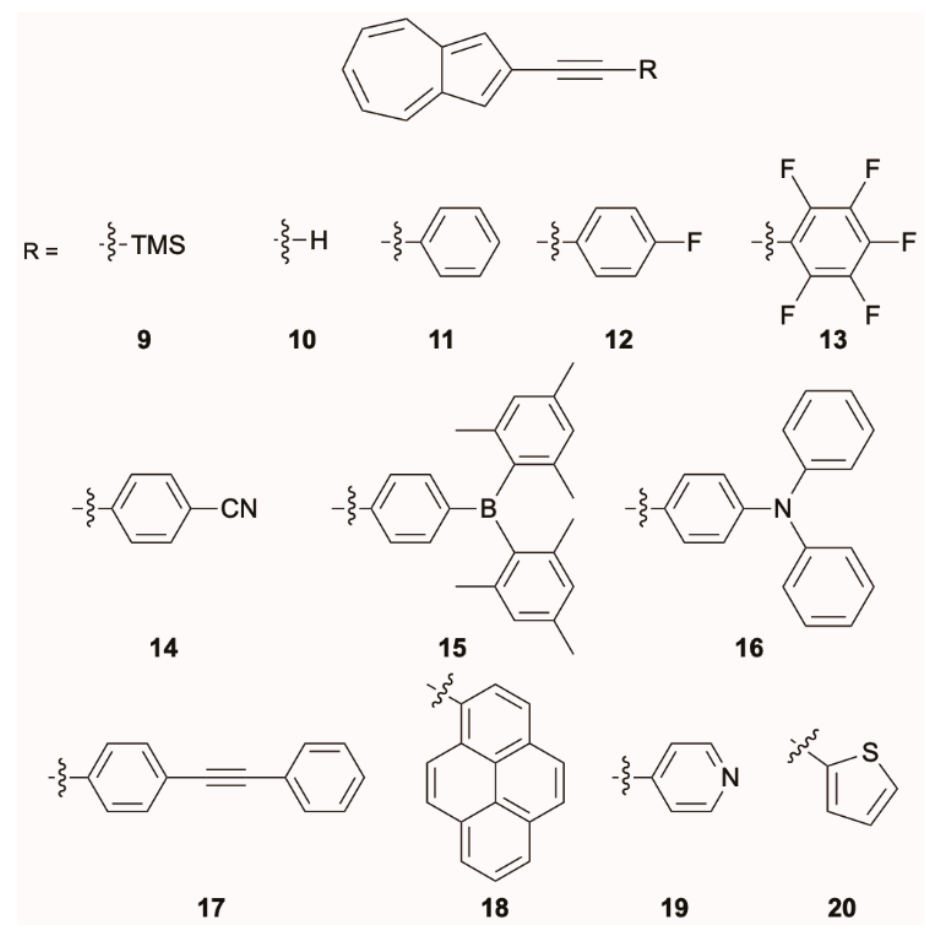

Scheme 7. Chemical structure of compounds 9-20.

Shoij et al. [43] reported on a family of azulene-fused phthalimides, among which derivative 21 (Scheme 8) displays the typical azulene fluorescent anti-Kasha feature. Compound 21 and a series of 2-arylazulenes reported by the same authors [44] show the typical intensification of the $S_{1}$ emission through protonation, leading to suppression of anti-Kasha behavior.<smiles></smiles>

Scheme 8. Chemical structure of compound 21.

In the search of new polycyclic heteroaromatics for potential optoelectronic applications, new examples of azulene derivatives showing anti-Kasha behavior were reported by Xin et al. [45], who prepared the first two azulene-based BN-heteroaromatics, 22 (Scheme 9) and 23, by nitrogen aromatic borylation. Their analysis was supported by TDDFT calculations at the CAM-B3LYP/Def2-TZVP level of theory. The same research group [46] described the reductive cyclization of 1-nitroazulene precursors to produce a series of azulene-pyridine-fused heteroaromatics, 24-29, displaying photophysical features typical of azulene derivatives with a weak emission band originating, based on CAM-B3LYP /6$311 G(d, p)$ TDDFT calculations, from $\mathrm{S}_{2}$ or higher excited states and no detectable emission from $S_{1}$. 


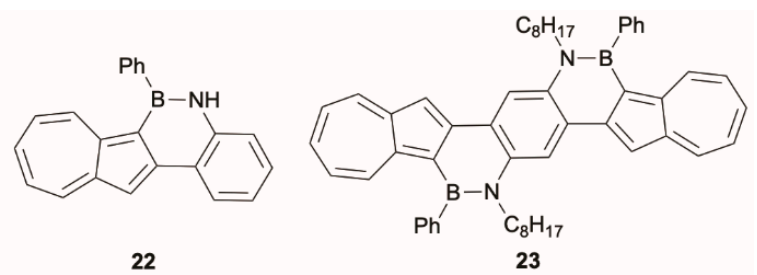

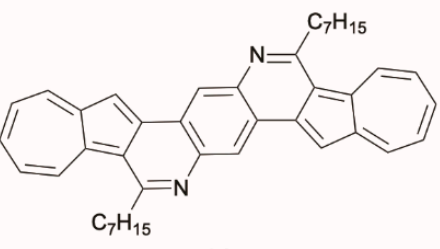

24
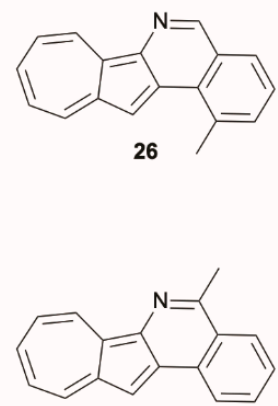

28<smiles>c1ccc2cc3ccccc3c-2cc1</smiles>

25<smiles></smiles>

27

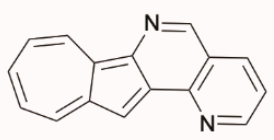

29

Scheme 9. Chemical structure of compounds 22-29.

\section{Cyclic Triimidazoles}

Lucenti et al. [47] investigated an extensive series of "cyclic triimidazole", TT (Scheme 10), derivatives (where TT is triimidazo[1,2-a:1', $\left.2^{\prime}-c: 1^{\prime \prime}, 2^{\prime \prime}-e\right][1,3,5]$ triazine) characterized, mainly in the solid state, by an intricated multiemissive behavior of molecular and aggregationinduced origin, including RTP associated with $\pi-\pi$ interactions. Within this rich photophysics, for some members of this family, a fluorescent or phosphorescent anti-Kasha behavior was recognized.

In particular, benzo[4,5]imidazo[1,2-a]benzo[4,5]imidazo[1,2-c]benzo[4,5]imidazo[1,2e] [1,3,5]triazine, 30 (Scheme 10), displays a single fluorescent emission at about $330 \mathrm{~nm}$ $(\Phi=17 \%)$ in diluted DCM solution at RT, which, based on TDDFT calculations at the $\omega B$ 97X $/ 6-311++G(d, p)$ level of theory, was associated with deactivation from an $S_{2}$ excited state [48]. This attribution was supported by a calculated substantial $S_{2}-S_{1}$ energy gap $(0.35 \mathrm{eV})$ and an $\mathrm{S}_{0}-\mathrm{S}_{1}$ transition with zero oscillator strength owing to the high symmetry (ideally $C_{3 \mathrm{~h}}$ ) of the molecular $\pi$-electron system. Intriguingly, solid 30 shows both phosphorescence and dual fluorescence $(\Phi=18 \%)$ at RT, which can be selectively activated by varying the excitation wavelength (see Figure 6). At $260 \mathrm{~nm}$ excitation wavelength, a near-UV fluorescence emission at $350 \mathrm{~nm}$ of $\mathrm{S}_{2}-\mathrm{S}_{0}$ origin, similar to that of the chromophore in dilute solution, is observed. By exciting at $370 \mathrm{~nm}$, a structured blue fluorescence at $407 \mathrm{~nm}$ superimposed onto the phosphorescent emission at $530 \mathrm{~nm}$ is produced. The authors explained the presence of this second fluorescence of $S_{1}-S_{0}$ nature, selectively activated only by populating the $S_{1}$ state, by partial loss of the molecular symmetry of the chromophore owing to interchromophoric interactions in the solid state. 
<smiles>c1cn2c(n1)n1ccnc1n1ccnc21</smiles>

Cyclic Triimidazole, TT

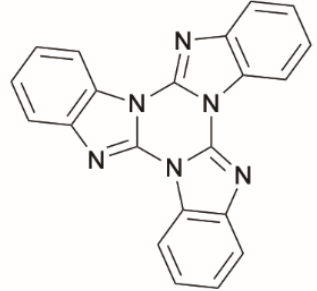

30<smiles>Cc1cnc2n3ccnc3n3ccnc3n12</smiles>

31<smiles>Clc1cnc2n3ccnc3n3c(Cl)cnc3n12</smiles>

32<smiles>Brc1cnc2n3ccnc3n3c(Br)cnc3n12</smiles>

35<smiles>Clc1cnc2n1c1ncc(Cl)n1c1ncc(Cl)n21</smiles>

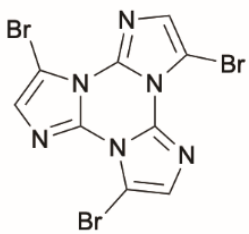

36<smiles>Brc1cnc2n3ccnc3n3ccnc3n12</smiles>

Scheme 10. Chemical structure of TT and compounds 30-37.
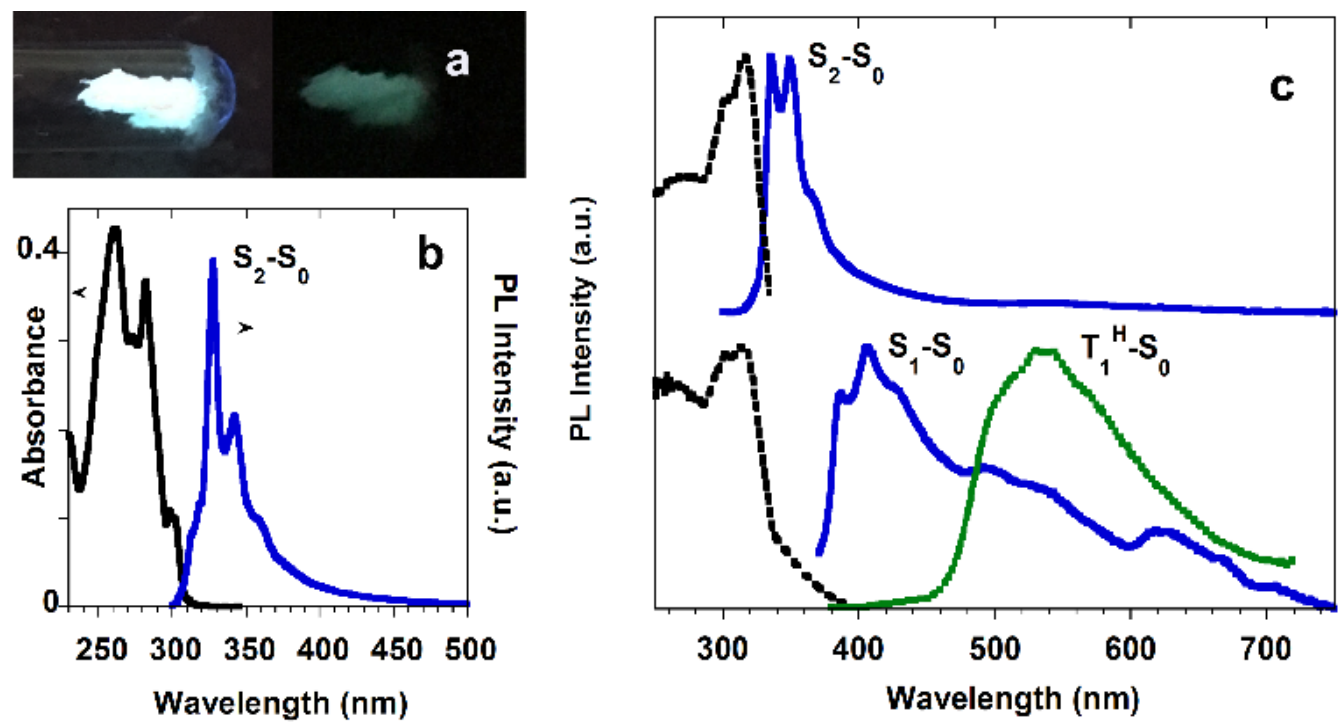

Figure 6. Compound 30. (a) Powders at $77 \mathrm{~K}$ with UV irradiation on (left) and off (right). (b) In DCM at RT: absorption and emission (blue line, $\lambda_{\mathrm{ex}}=260 \mathrm{~nm}$ ). (c) Powders at RT: Top: excitation (dashed black line, $\lambda_{\mathrm{em}}=348 \mathrm{~nm}$ ) and emission (blue line, $\lambda_{\mathrm{ex}}=260 \mathrm{~nm}$ ). Bottom: excitation (dashed black line, $\lambda_{\mathrm{em}}=408 \mathrm{~nm}$ ), emission (blue line, $\lambda_{\mathrm{ex}}=370 \mathrm{~nm}$ ) and phosphorescence (green dotted line, $10 \mathrm{~ms}$ delay, window $\left.50 \mathrm{~ms}, \lambda_{\mathrm{ex}}=358 \mathrm{~nm}\right)$. Reprinted with permission from [48].

Similarly, mono-, di- and trihalogenated TT compounds [48-50], 31-36 (Scheme 10), display dual fluorescence in the solid state due to a large $S_{n}-S_{1}$ energy splitting and a 
low or zero oscillator strength of the $\mathrm{S}_{0}-\mathrm{S}_{1}$ transition. In particular, $31-33(\Phi=24 \%$, $12 \%$ and $9 \%$, respectively) are characterized by multiple long-lived emissive components (due to molecular, $\mathrm{T}_{1}$ and aggregated forms) in addition to dual fluorescence (one at about $340 \mathrm{~nm}$ for the three compounds and the other at about 350, 400 and $370 \mathrm{~nm}$ for 31, 32 and 33, respectively; see Figure 7), better resolved at low temperature [49]. The two fluorescences, based on analysis of excitation spectra showing a first absorption at $320-330 \mathrm{~nm}$ and a second absorption at $300 \mathrm{~nm}$, were assigned to deactivation from $\mathrm{S}_{1}$ and $S_{n}(n>1)$, respectively, with an experimental $S_{2}-S_{1}$ energy gap in the $0.27-0.38 \mathrm{eV}$ range. This anti-Kasha origin of the dual fluorescence was supported by $\omega B 97 X / 6-311++G(d, p)$ DFT /TDDFT calculations which provided an $S_{1}$ state of low oscillator strength $(f=0.013$, 0.009 and 0 for 31, 32 and 33, respectively) due to symmetry reasons and high-energy singlet levels with large oscillator strength $\left(S_{3}\right.$ and $\left.S_{4}, f>0.4\right)$.
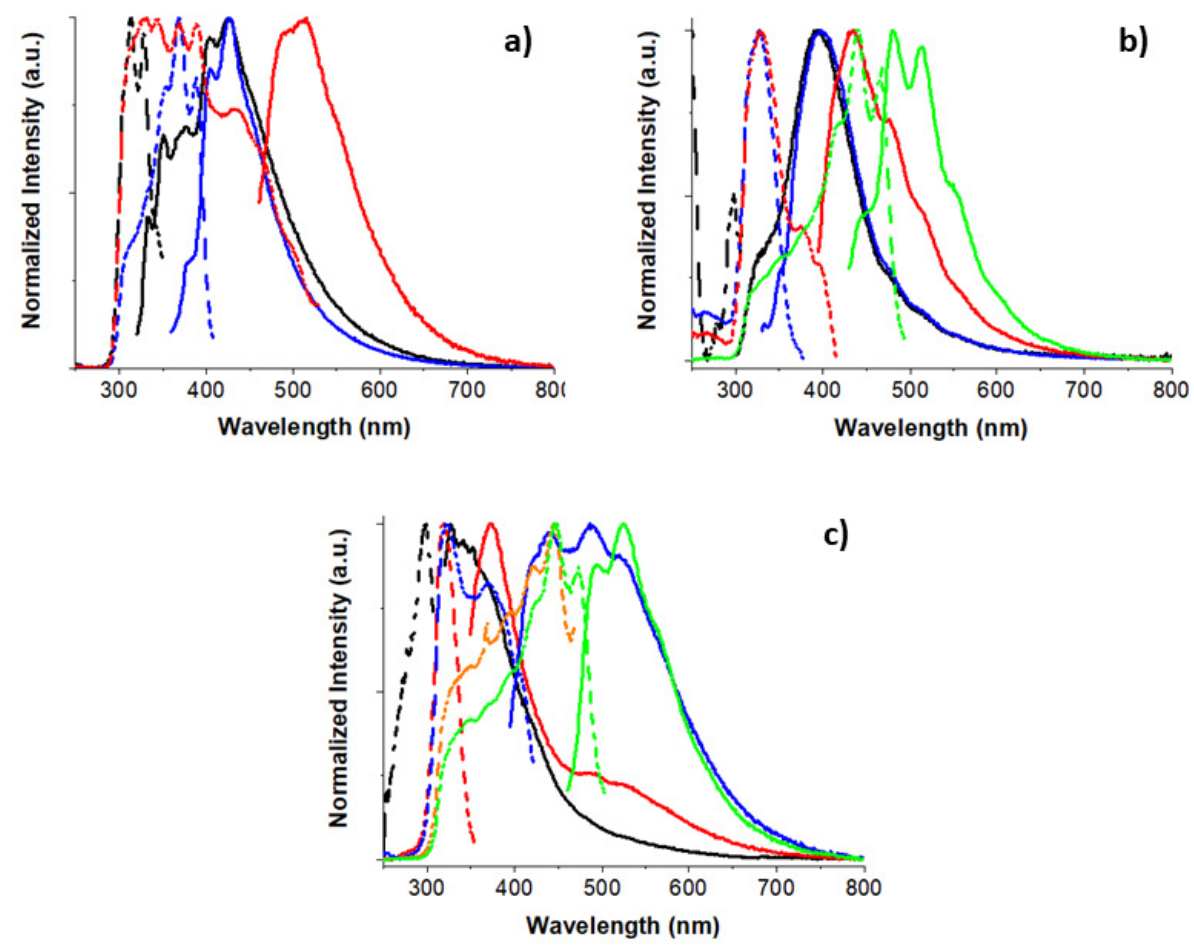

Figure 7. (a) Normalized excitation (dotted lines) and emission (solid lines) spectra of $\mathbf{3 1}$ crystals at $298 \mathrm{~K}$. Emission: $\lambda \mathrm{ex}=300 \mathrm{~nm}$ (black), $\lambda \mathrm{ex}=340 \mathrm{~nm}$ (blue) and $\lambda \mathrm{ex}=440 \mathrm{~nm}$ (red). Excitation: $\lambda \mathrm{em}=371 \mathrm{~nm}$ (black), $\lambda \mathrm{em}=427 \mathrm{~nm}$ (blue) and $\lambda \mathrm{em}=550 \mathrm{~nm}$ (red). (b) Normalized excitation (dotted lines) and emission (solid lines) spectra of 32 crystals at $298 \mathrm{~K}$. Emission: $\lambda \mathrm{ex}=280 \mathrm{~nm}$ (black), $\lambda e x=308 \mathrm{~nm}$ (blue), $\lambda \mathrm{ex}=375 \mathrm{~nm}$ (red) and $\lambda \mathrm{ex}=413 \mathrm{~nm}$ (green). Excitation: $\lambda \mathrm{em}=328 \mathrm{~nm}$ (black), $\lambda \mathrm{em}=398 \mathrm{~nm}$ (blue), $\lambda \mathrm{em}=436 \mathrm{~nm}$ (red) and $\lambda \mathrm{em}=514 \mathrm{~nm}$ (green). (c) Normalized excitation (dotted lines) and emission (solid lines) spectra of 33 crystals at $298 \mathrm{~K}$. Emission: $\lambda \mathrm{ex}=300 \mathrm{~nm}$ (black), $\lambda \mathrm{ex}=330 \mathrm{~nm}$ (red), $\lambda \mathrm{ex}=374 \mathrm{~nm}$ (blue) and $\lambda \mathrm{ex}=440 \mathrm{~nm}$ (green). Excitation: $\lambda \mathrm{em}=327 \mathrm{~nm}$ (black), $\lambda \mathrm{em}=373 \mathrm{~nm}$ (red), $\lambda \mathrm{em}=441 \mathrm{~nm}$ (blue), $\lambda \mathrm{em}=487 \mathrm{~nm}$ (orange) and $\lambda \mathrm{em}=524 \mathrm{~nm}$ (green). Adapted with permission from [49].

Analysis of the emissive properties of 31-33 in solution was precluded by the very low quantum efficiency of the compounds at low concentration. On the other side, DCM solutions of 34-36 [48,50] display a weak but recognizable fluorescence with maxima at 328,380 and $370 \mathrm{~nm}$, respectively ( $\Phi$ equal to $3 \%$ for 34 and almost vanishing for 35 and 36), which was ascribed, on the basis of $\omega B$ B7X/6-311++G(d,p) DFT/TDDFT calculations, to a high-energy $S_{n}$ state $(n=3 ; 4,5 ; 6,7$ for 34-36, respectively). Again, the absence of the $S_{1}-S_{0}$ radiative deactivation channel was explained by the low $f$ of the $S_{0}-S_{1}$ transition $(0.024,0.016$ and 0 for 34-36, respectively) associated with the high symmetry of the TT 
$\pi$-electron system, only partially disrupted in 34 and 35 , and to the sizable $S_{n}-S_{1}$ energy gap (calculated as $0.43,0.44$ and $0.48 \mathrm{eV}$ for $34-36$, respectively).

Interestingly, unlike $S_{1}$ and $S_{n}$ having $\left(\pi, \pi^{*}\right)$ character, the intermediate $S_{m}$ levels $\left(S_{2}\right.$, $S_{2,3}$ and $S_{2-4}$ for 34-36, respectively) correspond to forbidden transitions $(f=0)$ with partial $\left(\pi, \sigma^{*}\right)$ character, where the involved $\sigma$ orbitals are mainly delocalized on $\mathrm{Br}$ atom(s) and C-Br bond(s). Going to the solid state $(\Phi<0.1,14$ and $<0.1$ for 34-36, respectively) (see Figure 8 for 34), molecular and intermolecular-induced phosphorescences are observed in the spectra of the three compounds. Moreover, while an $\mathrm{S}_{\mathrm{m}}-\mathrm{S}_{0}$ emission is visible for 34-36 (at $326,345,365,382 ; 395,419,443 ; 394,418,444 \mathrm{~nm}$, respectively), the $S_{1}-S_{0}$ one appears only for $\mathbf{3 4}$ and $\mathbf{3 6}$ (with maxima at 426 and $415 \mathrm{~nm}$, respectively). This latter emission, associated with the distorting packing forces which reduce the symmetry of the molecular $\pi$-electron system, is the only emission observed by exciting at high energy $\left(E>S_{m}\right)$ while dual fluorescence is produced by exciting to $S_{m}$ levels. A possible interpretation was provided by $\omega$ B97X $/ 6-311++\mathrm{G}(\mathrm{d}, \mathrm{p}) \mathrm{DFT} / \mathrm{TDDFT}$ calculations on the $\pi-\pi$ dimer of 36 , which revealed a manifold of $S_{\mathrm{m}}$ levels of $\left(\pi, \pi^{*}\right),\left(\pi, \sigma^{*}\right)$ and mixed $\left(\pi, \pi^{*}\right) /\left(\pi, \sigma^{*}\right)$ character where the latter possess the strongest oscillator strength. It is therefore expected that excitation to $S_{m}$ results in both radiative deactivation (from $\left(\pi, \pi^{*}\right) /\left(\pi, \sigma^{*}\right)$ levels) to $S_{0}$ and IC (from $\left(\pi, \pi^{*}\right)$ levels) to $S_{1}$, while excitation to $\left(\pi, \pi^{*}\right) S_{n}$ leads to IC to $S_{1}$. In the case of 35 , no mixed $S_{m}\left(\pi, \pi^{*}\right) /\left(\pi, \sigma^{*}\right)$ states came out from calculations on its $\pi-\pi$ dimer, and the $(\pi$, $\left.\sigma^{*}\right)$ excitations have $f<0.01$, suggesting that $S_{\mathrm{m}}$ levels have predominant $\left(\pi, \pi^{*}\right)$ character with $f$ greater than that of $S_{1}$. Therefore, irrespective of excitation energy, these $\left(\pi, \pi^{*}\right) S_{m}$ levels are responsible for the observed single fluorescence.
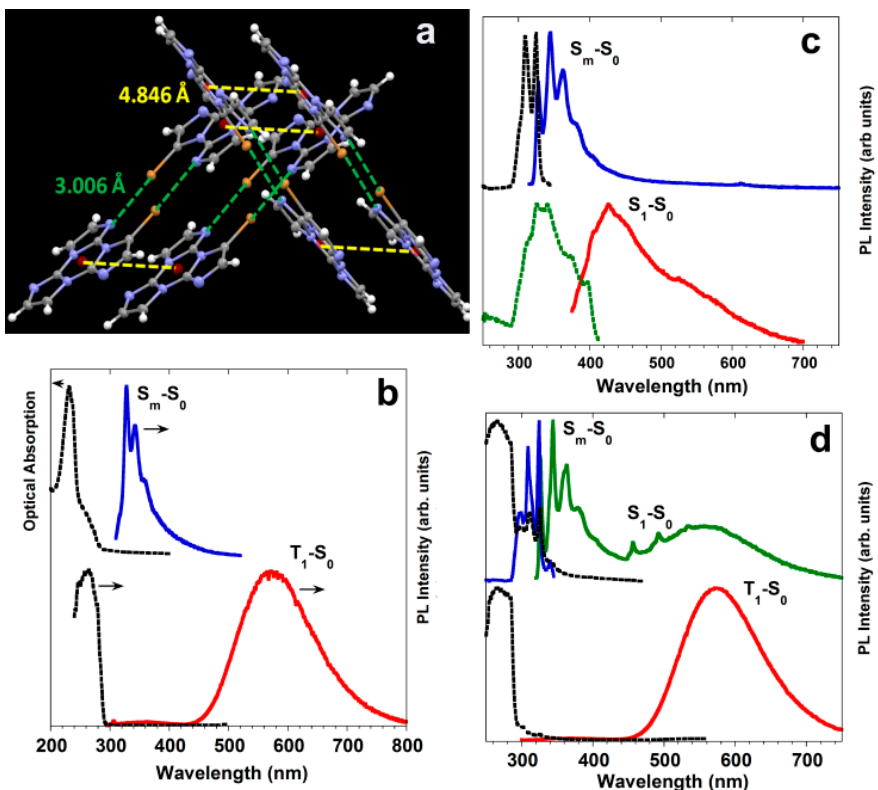

Figure 8. Compound 34. (a) Crystal packing: $\pi-\pi$ stacking interactions and $\mathrm{Br} \cdots \mathrm{N}$ XB shown as yellow and green dotted lines, respectively. (b) In DCM: top: absorption and emission ( $\lambda \mathrm{ex}=280 \mathrm{~nm})$ at RT; bottom: excitation (black dotted line, $\lambda \mathrm{em}=580 \mathrm{~nm}$ ) and emission (red line, $\lambda \mathrm{ex}=280 \mathrm{~nm}$ ) at $77 \mathrm{~K}$. (c) Powders at RT: top: excitation (black dotted line, $\lambda \mathrm{em}=363 \mathrm{~nm}$ ) and emission (blue line, $\lambda \mathrm{ex}=300 \mathrm{~nm}$ ); bottom: excitation (green dashed line, $\lambda \mathrm{em}=429 \mathrm{~nm}$ ) and emission (red line, $\lambda \mathrm{ex}=360 \mathrm{~nm}$ ). (d) Powders at $77 \mathrm{~K}$ : top: emission (green line, $\lambda \mathrm{ex}=300 \mathrm{~nm}$ ) and excitation (blue line, $\lambda$ em $=363 \mathrm{~nm}$; black dashed line, $\lambda \mathrm{em}=492 \mathrm{~nm}$ ); bottom: excitation (black dashed line, $\lambda \mathrm{em}=580 \mathrm{~nm}$ ) and emission (red line, $\lambda \mathrm{ex}=280 \mathrm{~nm}$ ). Reprinted with permission from [48].

3-(Pyridin-2-yl)triimidazo[1,2-a:1',2'-c:1", $\left.2^{\prime \prime}-e\right][1,3,5]$ triazine, 37 (Scheme 10), is the only member of the TT family showing anti-Kasha behavior from an excited triplet level [51]. This compound crystallizes in three phases characterized by a highly intricated photophysics comprising multiple fluorescent and phosphorescent bands $(\Phi=50 \%)$. 
The dual fluorescence observed at about 350 and $450 \mathrm{~nm}$ was associated with two different molecular conformations; therefore, it does not represent an exception to the Kasha rule. On the other side, two (at about 370 and $400 \mathrm{~nm}$ ) of the four observed phosphorescences were ascribed to molecular origin and interpreted, on the basis of $\omega B 97 X / 6-311++G(d, p)$ DFT/TDDFT calculations, as derived from triplet levels of different character. In particular, the high energy contribution resulted from the calculated $\mathrm{T}_{6}$ level having $\left(\sigma / \pi, \pi^{*}\right)$ character, while the low energy contribution derived from the calculated $\mathrm{T}_{1}$ with $\left(\pi, \pi^{*}\right)$ symmetry.

\section{1,2-Diphenylphenanthroimidazole (PPI) Derivatives}

1,2-Diphenylphenanthroimidazole, PPI (Scheme 11), represents an interesting tecton for the preparation of new emissive materials due to its intense blue fluorescence and its bipolar character associated with the presence of two distinct nitrogen atoms in the imidazole ring. Therefore, PPI can be used as an electron acceptor when combined with a strong electron-donor group or as an electron donor when combined with an electrondeficient or a weak electron-donor group. By exploiting PPI bipolarity, Yang et al. [52] prepared and investigated the symmetrical triads PPI-PCz, 38-41 (Scheme 11) (PCz is 9-phenylcarbazole), where PPI plays the role of the donor inside the molecule as revealed by femtosecond TA studies. Interestingly, for 38-41, anti-Kasha ICT behavior was reported.
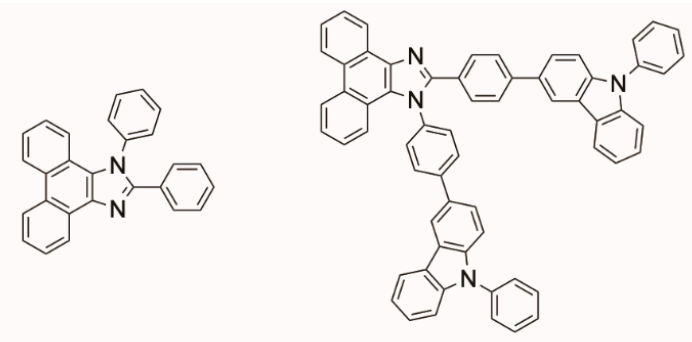

1,2-diphenylphenanthroimidazole, PPI

38

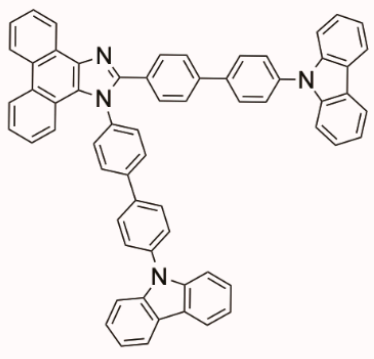

39

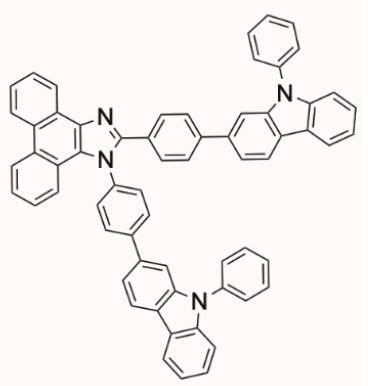

40

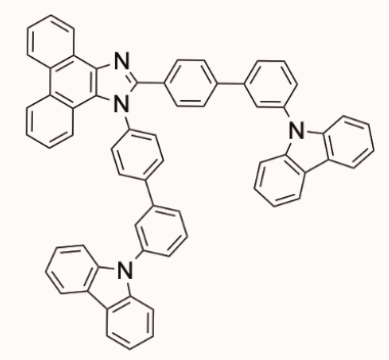

41

Scheme 11. Chemical structure of PPI and compounds 38-41. 
In diluted DMF solution, 38-41 display a fluorescent emission at $420 \mathrm{~nm}$ ( $\Phi$ almost $100 \%$ ), with a red shift of 55 and $30 \mathrm{~nm}$ with respect to that of PCz and PPI, respectively. TDDFT calculations on 38-41, performed at the B3LYP/6-311G(d,p) level of theory, revealed for the $S_{0}-S_{2}$ transition a higher probability than the $S_{0}-S_{1}$ one due to its higher oscillator strength. Moreover, calculations confirmed the $\mathrm{S}_{0}-\mathrm{S}_{2}$ ICT nature with the HOMO mainly located on the PPI fragment and the LUMO located on PCz and phenyl moieties.

Qiao et al. [53] reported on the excitation-dependent and anti-Kasha emissive behavior of 9-phenyl-10-(pyren-1-yl)-9H-pyreno[4,5- $d$ ] imidazole, $\mathbf{4 2}$ (Scheme 12).

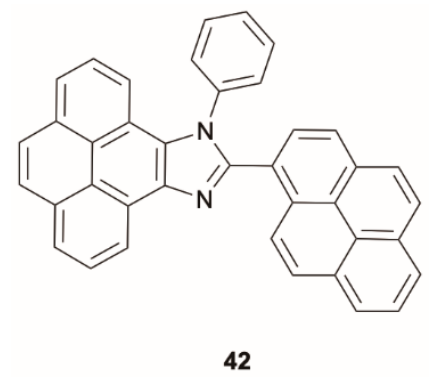

Scheme 12. Chemical structure of compound 42.

When excited at $350 \mathrm{~nm}$, neat and PMMA blended film of $\mathbf{4 2}$ display one fluorescence at $468 \mathrm{~nm}$ ( $\tau$ equal to $4.5 \mathrm{~ns}$ ). However, at $250 \mathrm{~nm}$ excitation wavelength, an additional dominant emission at $392 \mathrm{~nm}$ ( $\tau$ equal to $109.7 \mu \mathrm{s}$ ) is produced together with the $468 \mathrm{~nm}$ one. Excitation profiles of the two emissions obtained for the blended film indicated for the low-energy emission the same profile observed in absorption, while the high-energy emission is dominated by a sharp peak at around $250 \mathrm{~nm}$. Based on the experimental results and theoretical calculations, the emissive behavior was interpreted according to the mechanism reported in Figure 9. The anti-Kasha emission is attributed to the presence of a $\mathrm{T}_{\mathrm{n}}$ level easily populated through ISC from the high-energy singlet level $\left(\mathrm{S}_{5}\right)$. Subsequent ISC to $S_{2}$ results in the high-energy long-lived fluorescence. It should be noted that, for this system, a mixed DFT/TDDFT computational protocol has been adopted, where the $\mathrm{S}_{0}$ and $S_{1}$ geometries were optimized at B3LYP/6-31G $(d, p)$ level while the higher-energy singlet levels and the triplet states were calculated at M062X/6-31G(d,p) level on the optimized geometry of $S_{1}$.

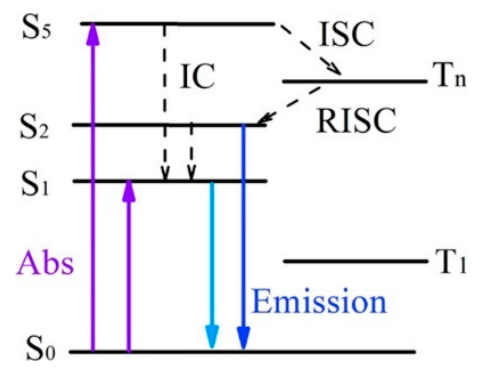

Figure 9. Exciton evolution route. Schematic potential key photophysical processes proposed for anti-Kasha photoemission of 42. Abs, IC, ISC and RISC represent the absorption, IC, ISC and reverse ISC, respectively. The absorption and emission processes are represented as solid arrow lines. The nonradiative processes are indicated as dashed arrow lines. Reprinted with permission from [53], Copyright 2019, American Chemical Society. 
Jiang et al. [54] reported the photophysical investigation of compound 43 (Scheme 13) in which PPI is connected to the anthracene-9-carbonitrile ( $\mathrm{AnCN}$ ) fragment. The absorption bands of the compound are a combination of the independent absorption bands of the PPI and AnCN units while the emission displays a solvatochromic behavior, consistent with its CT character. This was supported by DFT calculations which revealed that HOMO and LUMO are delocalized on the PPI and AnCN units, respectively, and are almost totally separated. PL spectra in toluene solution display an emission at $463 \mathrm{~nm}$ and a very weak one in the $360-400 \mathrm{~nm}$ range which becomes predominant when triethylamine is added to the solution (see Figure 10). Through comparative spectroscopical studies, the high-energy emission was associated with the PPI unit. Based on TDDFT calculations at the M06-2X/6$31 G(d, p)$ level (see Figure 11), the origin of the two emissions was ascribed to radiative decay from molecular $S_{4}$ and $S_{1}$ excited states. In particular, calculations indicated $S_{1}$ and $\mathrm{S}_{3}$ states localized on the AnCN unit, $\mathrm{S}_{2}$ state with CT character from PPI to AnCN and $\mathrm{S}_{4}$ and $\mathrm{S}_{5}$ both localized on PPI units. Anti-Kasha emission from $\mathrm{S}_{4}$ was explained by the poor electronic coupling between $S_{1}$ and $S_{4}$, the high $S_{4}-S_{1}$ energy gap $(0.75 \mathrm{eV})$ and the large oscillator strength of $S_{4}$ with respect to $S_{2}$ and $S_{3}$. In agreement with time-resolved analysis, quenching of the low-energy emission by triethylamine was ascribed to exciplex formation between the $S_{1}$ state and triethylamine, which results in an inhibited $S_{4}-S_{1}$ IC and therefore in an intensification of the high-energy emission.

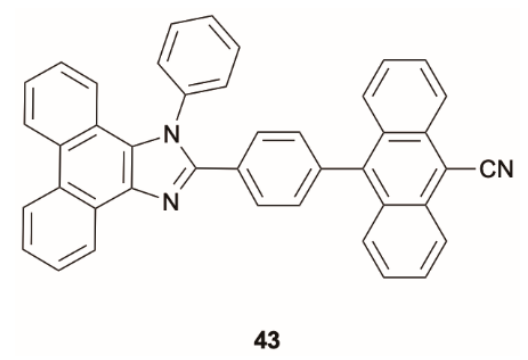

Scheme 13. Chemical structure of compound 43.

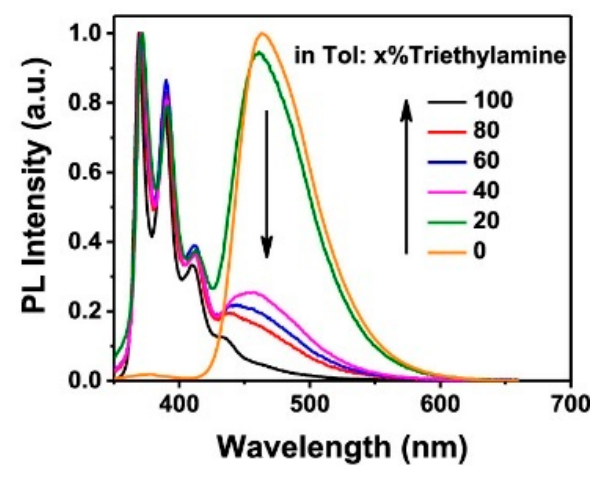

Figure 10. The normalized PL spectra of 43 in toluene and triethylamine mixed solution with different triethylamine fractions. Adapted with permission from [54], Copyright 2019, American Chemical Society. 
(a)
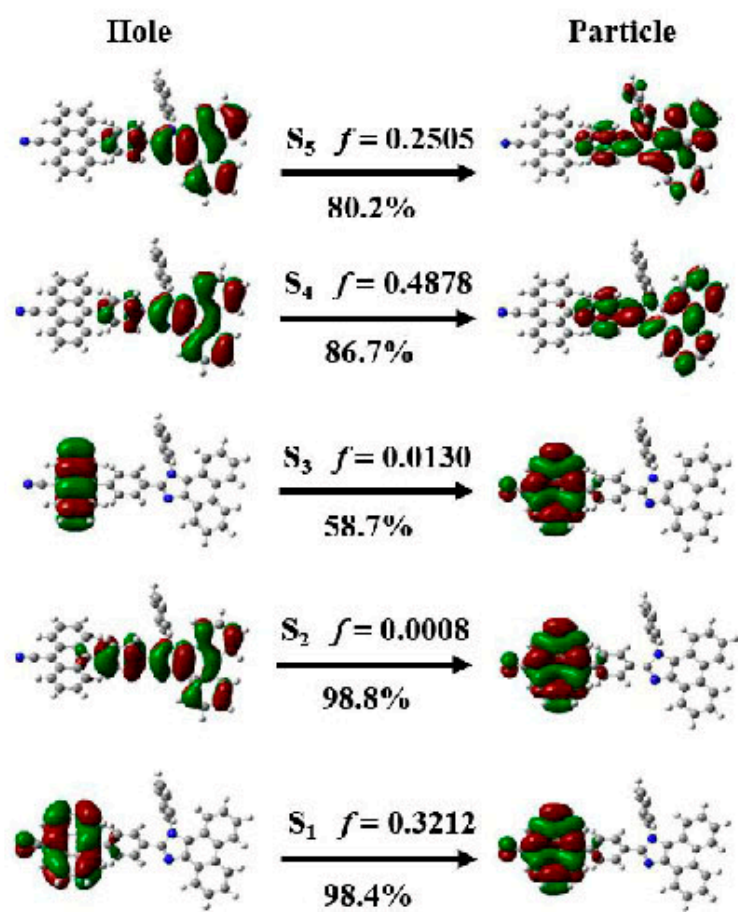

(b)

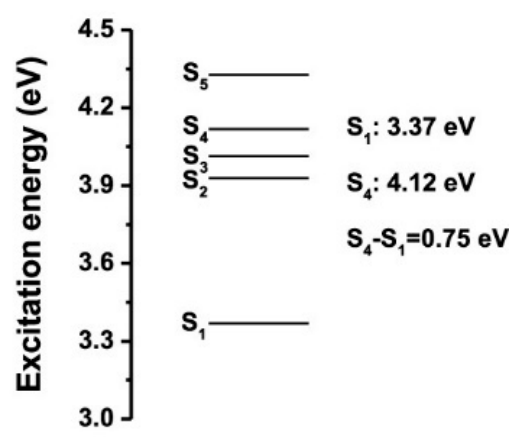

Figure 11. (a) Natural transition orbitals of 43; (b) the energy landscape for singlet states of 43. Reprinted/adapted with permission from [54], Copyright 2019, American Chemical Society.

\section{Cyanines}

The peculiar electronic structure of cyanines, having an odd number of conjugated $p_{z}$ orbitals and an even number of $\pi$ electrons, results in the stabilization of the $S_{1}$ state with respect to $S_{2}$. Therefore, frequently, these dyes are characterized by an intense $S_{0}-S_{1}$ transition in the visible/near-IR region and much less intense $S_{0}-S_{n}(n>1)$ transitions in the visible/near-UV region. Another relevant aspect of cyanine dyes is their large two-photon absorption cross-sections for near-IR wavelengths. These peculiar properties of cyanine dyes have attracted much interest in many applications such as two-photon photosensitizer or antenna systems. In addition, the large $S_{2}-S_{1}$ energy gaps $(0.6-1.0 \mathrm{eV})$ associated with low $S_{2}-S_{1}$ IC rates represent an interesting feature for possible anti-Kasha behavior. In fact, some cyanine dyes exhibit dual fluorescence when excited directly to $S_{2}$, as reported by Das et al. [55] in 2013. In particular, cyanine IR125 (44) (Scheme 14) in DMSO produces a single fluorescence at $851 \mathrm{~nm}$ when excited at $805 \mathrm{~nm}$, but a second fluorescence at $571 \mathrm{~nm}$ (of $\mathrm{S}_{2}-\mathrm{S}_{0}$ origin) is activated together with the one at $851 \mathrm{~nm}$ (of $\mathrm{S}_{1}-\mathrm{S}_{0}$ nature) upon excitation at $527 \mathrm{~nm}$. According to steady-state and time-resolved experiments, dual fluorescence was schematized as reported in Figure 12. By high-energy excitation, direct fluorescence from $S_{2}$ is produced together with IC to $S_{1}$, from which fluorescence is also observed. By low-energy excitation, only $S_{1}$ is populated, therefore resulting in single fluorescence. 

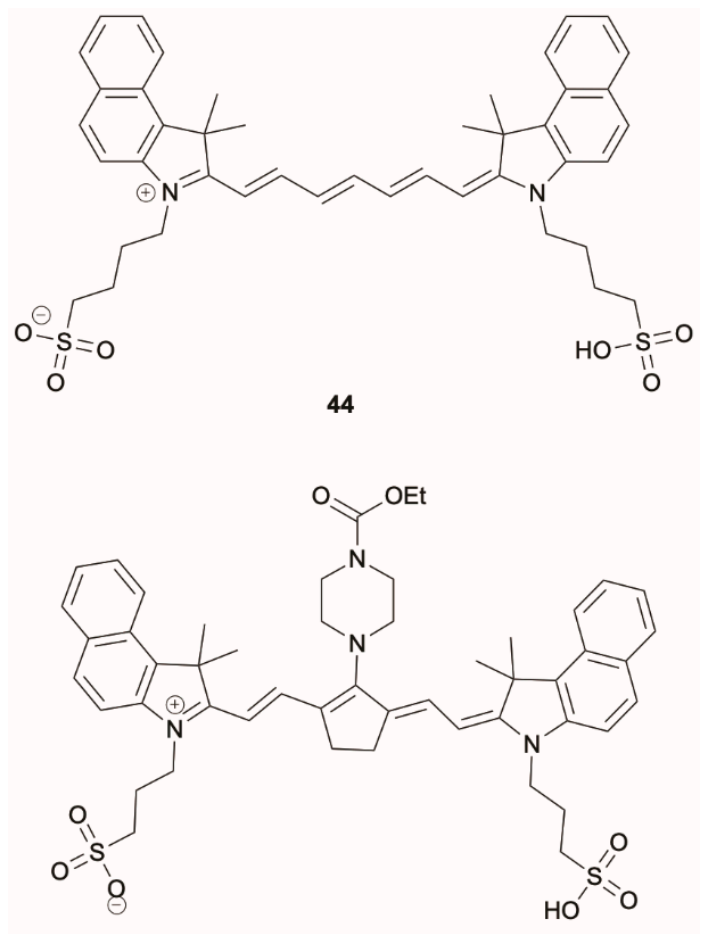

45<smiles>CCN1/C(=C/C=C2\CCC(/C=C/c3sc4ccc(Cl)cc4[n+]3CC)=C2N(c2ccccc2)c2ccccc2)Sc2ccc(Cl)cc21</smiles>

46

Scheme 14. Chemical structure of compounds $44-46$.

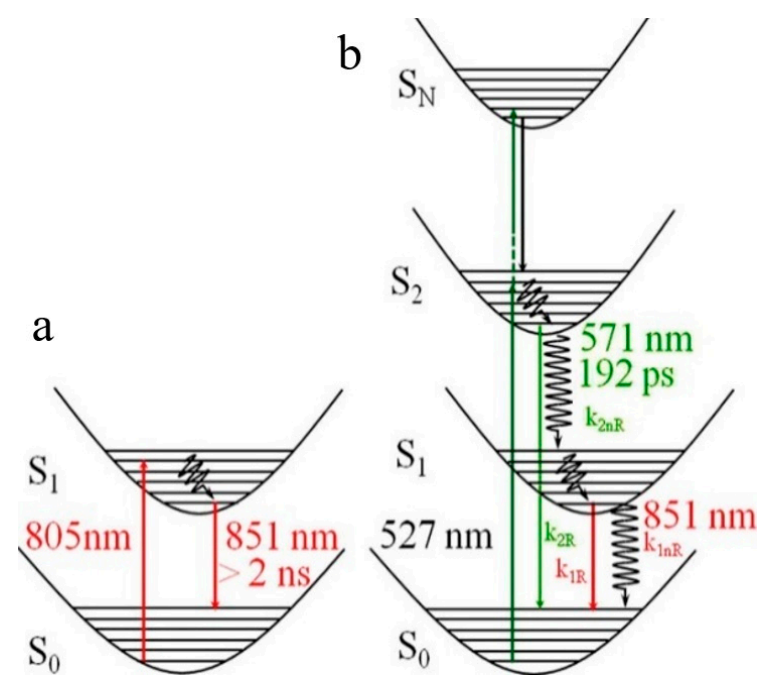

Figure 12. Schematic diagram of excitation of IR125 (44) in DMSO by (a) $805 \mathrm{~nm}$ laser pulse (lowenergy excitation) and (b) $527 \mathrm{~nm}$ laser pulse (high-energy excitation). Incident laser polarization dependent studies have shown the importance of higher excited states in the fluorescence emission as discussed in the text. Reprinted from [55], Copyright 2013, with permission from Elsevier. 
Later on, Das et al. [56] reported two near-infrared (NIR) tricarbocyanine dyes, IR144 and IR140 (45 and 46, respectively) (Scheme 14), displaying two distinct fluorescent emissions in different alcoholic solutions $(\mathrm{MeOH}, \mathrm{EtOH}, \mathrm{PrOH}$ and $\mathrm{BuOH})$ : one in the visible region (500-550 nm), assigned to an $\mathrm{S}_{2}-\mathrm{S}_{0}$ transition, and the other in the NIR (850-900 nm), assigned to an $S_{1}-S_{0}$ transition. For both 45 and 46 , a variation of the $S_{2}$ emission intensity and lifetimes by varying the alcohol chain length was observed. Differently from 46,45 displays an $\mathrm{S}_{2}$ excited state dynamics which depends on the solvent polarizability as predicted by the energy gap law. By increasing the polarizability of the alcohol series (from $\mathrm{MeOH}$ to $\mathrm{BuOH}$ ), $\mathrm{S}_{2}$ emission lifetimes of 45 can be extended from a few picoseconds to quite larger values (83.46 ps in $\mathrm{BuOH}$ ), opening up a new area for cell imaging and tissue engineering in different alcohols.

Nairat et al. [57] proposed an original way to modulate the Kasha rule through photonic control in cyanine dyes by using shaped pulses for direct $S_{2}$ excitation. The authors tracked the $S_{2}$ and $S_{1}$ fluorescence yield for different cyanine dyes, i.e., IR144 (45) and mPi-IR806 (47) (Scheme 15), in solution as a function of the femtosecond pulse produced by a linear chirp device. These findings offer a photonic method to control $\mathrm{S}_{2}$ population, allowing the exploration of photochemical processes initiated from higher excited states.

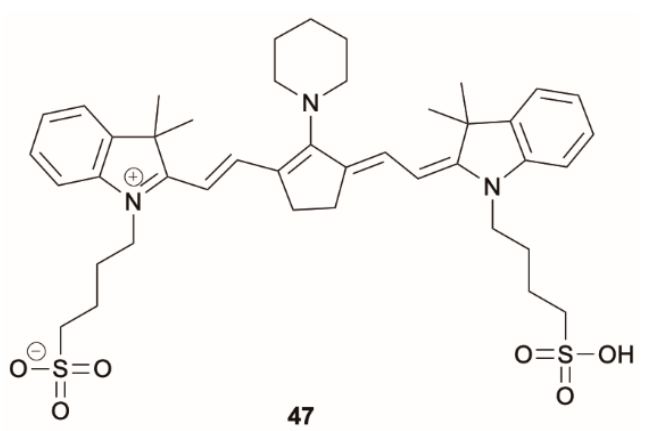

Scheme 15. Chemical structure of compound 47.

Guarin et al. [58] reported direct measurements of the $S_{2}$ lifetime for three cyanine families with benzothiazolyl (48-50) (Scheme 16), 2-quinolyl (51-53) (Scheme 16) and 4quinolyl (54-56) (Scheme 16) end groups and different $-(C=C)_{n}-$ skeleton sizes $(n=1,2$ and 3 for each family) in order to investigate the structural features able to slow down or inhibit $S_{2}-S_{1}$ IC. Clear emission peaks in the region between the first and second absorption transitions, ascribable to emission from $S_{2}$, were detected for the longer cyanines in ethanol solution (at 429 and $494 \mathrm{~nm}$ for 49 and 50, respectively; at about 460 and $490 \mathrm{~nm}$ for $\mathbf{5 2}$ and 53, respectively; at 460 and $523 \mathrm{~nm}$ for 55 and 56, respectively), while the shortest ones displayed weak emission from $\mathrm{S}_{2}$ only in more concentrated solutions. The dynamics of the upper states was followed by femtosecond fluorescence upconversion and by time-resolving spontaneous emission. Moreover, the time evolution of the $\mathrm{S}_{1}$ emission allowed the monitoring of the population growth of $S_{1}$ through IC from $S_{2}$. The authors observed that $S_{2}$ lifetime can vary by more than two orders of magnitude depending on the conjugation length and end groups. For the shortest cyanines, $\mathbf{5 1}$ and $\mathbf{5 4}$, ultrafast $(<0.4 \mathrm{ps}) \mathrm{S}_{2}$ lifetimes were measured, with a correspondingly fast $\mathrm{S}_{1}$ population. These rapid dynamics were associated with the nonplanar ground state geometry due to steric hindrance between the end groups. For all the other cyanines (48-50, 52, 53, 55 and 56), having planar structures, the $S_{2}$ lifetimes were found to closely follow the energy gap law, so that the cyanine with the largest gap, 50, displays the slowest (17.3 ps in ethanol) $S_{2}$ decay time among all the analyzed compounds. 


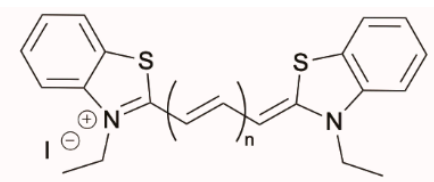

3,3'-diethyl-2,2'-thiacarbocyanine idodide , 48

3,3'-diethyl-2,2'-thiadicarbocyanine iodide, 49

3,3'-diethyl-2,2'-thiatricarbocyanine idodide , 50

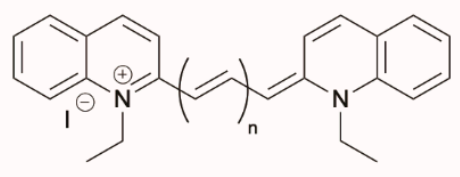

1,1'-diethyl-2,2'-cyanine iodide, 51

1,1'-diethyl-2,2'-carbocyanine iodide, 52

1,1'-diethyl-2,2'-dicarbocyanine iodide, 53

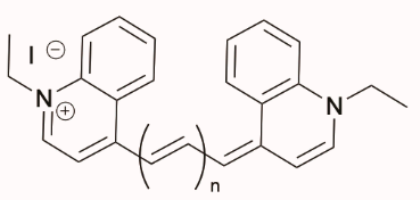

1,1'-diethyl-4,4'-cyanine iodide, 54

1,1'-diethyl-4,4'-carbocyanine iodide, 55

1,1'-diethyl-4,4'-dicarbocyanine iodide, 56

Scheme 16. Chemical structure of compounds $\mathbf{4 8 - 5 6 .}$

Similarly, Kumari and Gupta [59] showed that indocyanine green ICG, 57 (Scheme 17), an exogenous contrast agent used for clinical applications in the NIR region for its $810 \mathrm{~nm}$ emission from $S_{1}$, displays direct fluorescence at $572 \mathrm{~nm}$ from $S_{2}$ when excited at $393 \mathrm{~nm}$. $S_{2}$ level can be populated also by two-photon excitation with a $790 \mathrm{~nm}$ wavelength femtosecond laser, in the well-known "tissue-optical window", providing a two-photon contrast agent for biomedical imaging applications. The authors demonstrated the use of $\mathbf{5 7}$ as exogenous contrast agent for HeLa cell imaging, exploiting its direct emission from the $\mathrm{S}_{2}$ state.

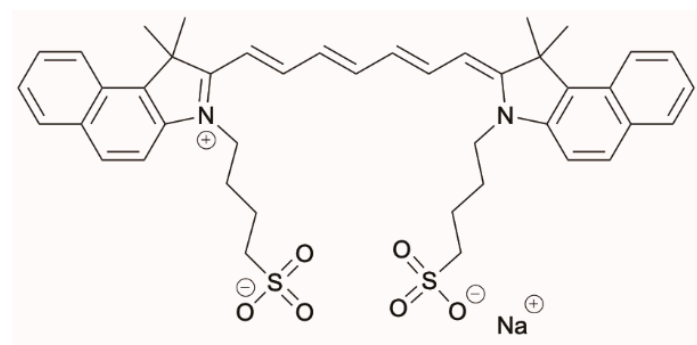

57

Scheme 17. Chemical structure of compound 57.

\section{Thiophenes}

Thiophene-based compounds are potential candidates to display anti-Kasha phosphorescence from $\mathrm{T}_{2}$ since thienyl substituents have been reported, through a combined 
spectroscopic and B3LYP/6-31G(d) DFT and TDDFT investigation, to favor $\mathrm{S}_{1}$ to energymatched $\mathrm{T}_{2}$ ISC [60].

Chaudhuri et al. [61] prepared and studied compound 58 (Scheme 18), which has a thiophene-decorated phenazine linked to a triphenylene block. Compound 58 displays in solution one fluorescent emission at $630 \mathrm{~nm}$ corresponding to deactivation from $\mathrm{S}_{1}$. Concomitant phosphorescent emission at $760 \mathrm{~nm}$ at room temperature becomes visible only in polystyrene matrix with exclusion of oxygen (see Figure 13). Intriguingly, room temperature EL spectra of 58 in poly(9-vinylcarbazole) matrix display fluorescence/phosphorescence dual EL (see Figure 14). Moreover, EL and gated spectra of diluted polystyrene films, collected at 4 and $25 \mathrm{~K}$, respectively, show an additional peak at $530 \mathrm{~nm}$ from a high-energy triplet. Based on PBE0/6-31G(d) geometry optimization and PBE0/6-311G(d) TDDFT calculations on the analog compound with the long alkyl chains (R) replaced by methyl groups, the two phosphorescences were assigned to triplet states localized on the triphenylene (the high-energy emission) and the phenazine moieties (the low-energy one). At RT, the high-energy triplet emission is quenched by vibrations that favor IC from the "hot" triphenylene triplet to the lower-energy phenazine one. It was therefore suggested that anti-Kasha dual phosphorescence may favor the realization of room-temperature electrophosphorescent devices.

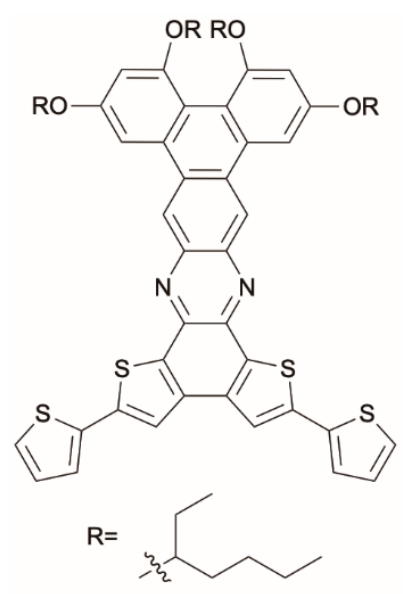

58

Scheme 18. Chemical structure of compound 58.

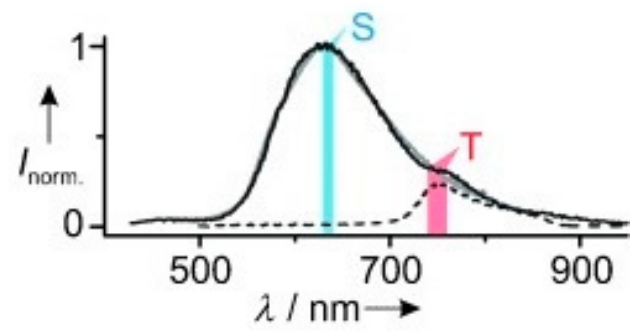

Figure 13. OLED EL spectra of 58 compared to solution PL (gray line) and delayed PL from dilute dispersions in polystyrene films (dashed line). The delayed PL is measured in vacuo to prevent triplet quenching. Adapted with permission from [61]. 


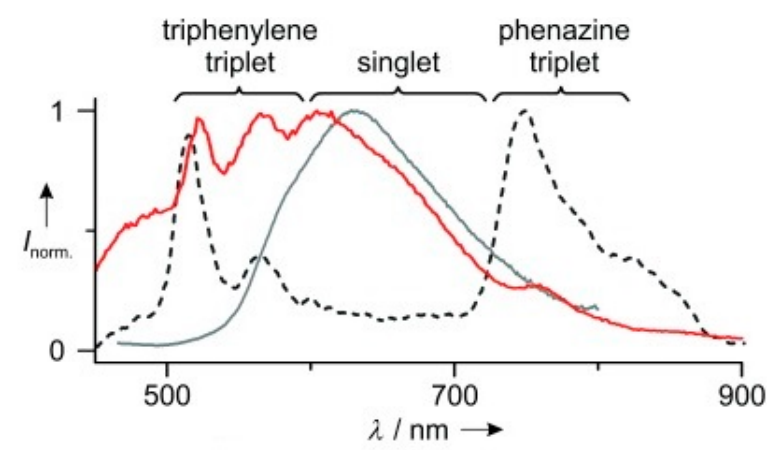

Figure 14. Electrically and optically excited phosphorescence from high-lying triplets of 58. EL spectrum at $4 \mathrm{~K}$ (red), room-temperature solution PL (gray) and gated dilute-film PL (25 K, dashed). Two dominant distinct phosphorescence peaks are identified. Adapted with permission from [61].

He et al. [62] proposed a design strategy to develop pure-phosphorescent singlemolecule white light emitters by incorporating benzophenone derivatives into the dibenzothiophene unit. By Friedel-Crafts acylation, five compounds, namely dibenzo[ $[b, d]$ thiophen-2-yl(phenyl)methanone (59), dibenzo[ $b, d]$ thiophen-2-yl(4-fluorophenyl)methanone (60), dibenzo[ $[b, d]$ thiophen-2-yl(4-chlorophenyl)methanone (61), dibenzo $[b, d]$ thiophen-2-yl(4bromophenyl)methanone (62) and 4-bromo-4'-chlorobenzophenone (63) (Scheme 19) were synthesized, for which phosphorescence was expected to be triggered by the presence of both the carbonyl group and the heavy atom. Moreover, the dibenzothiophene moiety should favor dual phosphorescence by providing various triplet excited states with different molecular orbital configurations. The derivatives display CIE behavior showing weak luminescence in solution and intense emission as crystals at ambient conditions arising from two phosphorescent contributions: a fast one at $470 \mathrm{~nm}(\tau=0.06-0.71 \mathrm{~ms})$, which has been attributed to a radiative decay from $\mathrm{T}_{2}$, and a long-lived one at about $570 \mathrm{~nm}$ $\left(\tau=104-123 \mathrm{~ms}\right.$ ) due to emission from $\mathrm{T}_{1}$ (see Figure 15). The assignment of the fast decay to a phosphorescence rather than a TADF was supported by low-temperature measurements, revealing an increase in lifetime with a decrease in temperature. Moreover, quantum mechanics/molecular mechanics (QM/MM) studies on selected compounds, where the QM region was treated at B3LYP/6-31G(d) level for both DFT and TDDFT calculations, provided two lowest excited states $\left(T_{1}\right.$ and $\left.T_{2}\right)$ below $S_{1}$, with large $S_{1}-T_{1}$ separation (in the $0.72-0.88 \mathrm{eV}$ range), suggesting that TADF is less likely to occur at RT. The key point for successfully obtaining dual phosphorescence relies on the phenyl group shared between benzophenone and dibenzothiophene moieties providing, respectively, $\left(n, \pi^{*}\right)$ and $\left(\pi, \pi^{*}\right)$ character to the transitions. As a result, although both $\mathrm{T}_{1}$ and $\mathrm{T}_{2}$ states have mixed $\left(n, \pi^{*}\right)$ and $\left(\pi, \pi^{*}\right)$ configuration, $\mathrm{T}_{2}$ is mainly an $\left(n, \pi^{*}\right)$ transition while $\mathrm{T}_{1}$ possesses more $\left(\pi, \pi^{*}\right)$ character. According to the El-Sayed rule, a larger SOC is expected for $T_{2}-S_{0}$ than for $T_{1}-S_{0}$. This leads to a short lifetime for $T_{2}$ and a long lifetime for $T_{1}$. The anti-Kasha emission might then occur after the thermal population of $\mathrm{T}_{2}$ from $\mathrm{T}_{1}$ due to a small $\Delta \mathrm{E}\left(\mathrm{T}_{1}-\mathrm{T}_{2}\right)(0.19$ and $0.27 \mathrm{eV}$ from experiment and theory, respectively, for 61 ), comparable to RT thermal energy.

Later on, Paul et al. [63] applied computational techniques to re-examine the previously reported anti-Kasha behavior of $\mathbf{6 1}$ [62]. According to the new calculations, performed by DFT and TDDFT approaches at the Grimme's dispersion corrected B3LYP /6$311 \mathrm{G}(\mathrm{d}, \mathrm{p})$ level of theory, the $\Delta \mathrm{E}\left(\mathrm{T}_{1}-\mathrm{T}_{2}\right), 0.48 \mathrm{eV}$, is too large to obtain a sufficient Boltzmann population of $T_{2}$ from $T_{1}$. Therefore, they proposed that $T_{2}$ is instead populated by $S_{1}-T_{2}$ ISC. These two levels are in fact separated by only $0.09 \mathrm{eV}$ and are characterized by strong Duschinsky mixing [64] between their normal modes. 
<smiles>O=C(c1ccccc1)c1ccc2sc3ccccc3c2c1</smiles>

59<smiles>O=C(c1ccc(F)cc1)c1ccc2sc3ccccc3c2c1</smiles>

60<smiles>O=C(c1ccc(Cl)cc1)c1ccc2sc3ccccc3c2c1</smiles>

61

62<smiles>O=C(c1ccc(Cl)cc1)c1ccc(Br)cc1</smiles>

63

Scheme 19. Chemical structure of compounds 59-63.

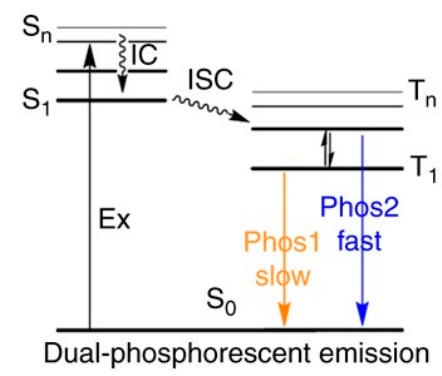

Figure 15. Jablonski diagram for dual phosphorescent emission of 59-63. Adapted with permission from [62]. Copyright 2017, Springer Nature.

Cao et al. [65] prepared and investigated a series of highly planar indaceno[1,2-b:5,6$b^{\prime}$ ]dithiophene 1,6-dioxide derivatives containing out-of-plane side chains aimed at preventing quenching of the emission due to strong intermolecular $\pi-\pi$ interactions. Among the studied compounds, film of 64 (Scheme 20) displays anti-Kasha phosphorescence.

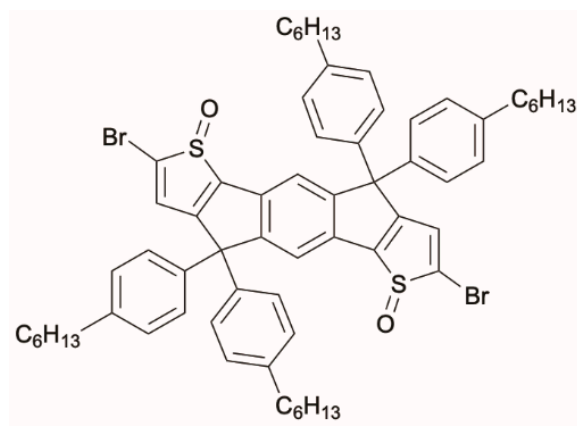

64

Scheme 20. Chemical structure of compound 64.

Diluted chloroform solution of 64 displays, when excited at $380 \mathrm{~nm}$, a vibrationally resolved emission originating from a locally excited state at 400-500 $\mathrm{nm}$ and an additional broad band of ICT character at 500-700 nm. The film of 64 shows excitation-dependent photoluminescence. In particular, upon excitation at $340 \mathrm{~nm}$, a fluorescent band at $570 \mathrm{~nm}$ 
appears in the spectrum. While, at $253 \mathrm{~nm}$ excitation, dual emission comprising a phosphorescence at $400 \mathrm{~nm}(\tau=0.102 \mathrm{~ms})$ and a fluorescence at $570 \mathrm{~nm}(\tau=0.812 \mathrm{~ns})$ is observed. The phosphorescence was attributed to anti-Kasha deactivation originating from a highenergy triplet level. In agreement, DFT-TDDFT calculations on a model molecule where hexyl was replaced by methyl, performed at B3LYP/6-31G(d) level for $S_{0}$ and $S_{1}$ and at M06-2X/6-31G(d) level for higher singlet states and for triplet states, revealed small $S_{2-3}-T_{3-4}$ energy gaps and a large $T_{3}-T_{2}$ one. This justifies an easy ISC to populate high triplet levels from which phosphorescence is observed due to inhibited IC. Therefore, it was suggested that upon excitation to $S_{1}$, only the corresponding fluorescence at $570 \mathrm{~nm}$ is observed. However, by populating upper excited states $\left(S_{n}\right)$, relaxed $S_{1}$ (by IC) and $\mathrm{T}_{\mathrm{n}}$ (by ISC) are obtained, from which fluorescence and anti-Kasha phosphorescence are respectively produced (see Figure 16).

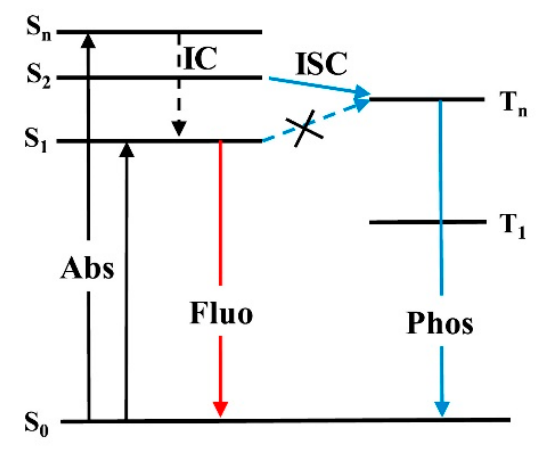

Figure 16. The proposed mechanism for the anti-Kasha properties of $\mathbf{6 4}$ film. Reprinted from [65], Copyright 2020, with permission from Elsevier.

\section{Carbazoles}

Carbazole possesses a high triplet energy level which induces a small energy gap between triplet and singlet states beneficial for ISC [66]. Feng et al. [67] developed a fully organic compound, 65 (Scheme 21) (containing carbazole $(\mathrm{Cz})$, a carbonyl group $(\mathrm{Cb})$ and dibenzothiophene (DBT)), able to display in DCM solution RTP from $\mathrm{T}_{2}$ (at $485 \mathrm{~nm}$ ) with $\Phi$ varying from $25 \%$ in air to $40 \%$ under nitrogen. The ICT features of such emission were proven by a significant solvatochromic red shift with increasing solvent polarity. Experimental studies performed at $77 \mathrm{~K}$ revealed for the molecule three radiative deactivation paths assigned to $S_{1}, T_{2}$ and $T_{1}$, with bands respectively at 440,465 and $570 \mathrm{~nm}$, originating from the same photogenerated excited state. Based on the observation that, among the three bands, that associated with $\mathrm{T}_{2}$ is the strongest while that originating from $T_{1}$ is the weakest and has the longest lifetimes, an easy $S_{1}-T_{2}$ ISC and a slow $T_{2}-T_{1}$ IC were suggested. Such interpretation was supported by B3LYP/6-31G(d) DFT and TDDFT calculations in DCM, which provided two lowest triplet excited states, $\mathrm{T}_{1}$ and $\mathrm{T}_{2}$, both of mixed $\left(n, \pi^{*}\right)$ and $\left(\pi, \pi^{*}\right)$ character, below the $\left(\pi, \pi^{*}\right) \mathrm{S}_{1}$ level, with $\Delta \mathrm{E}_{\mathrm{ST}}$ energy gaps equal to 0.305 and $0.04 \mathrm{eV}$, respectively. While $\mathrm{T}_{1}$, mainly localized on the $\mathrm{Cz}$ unit, has a predominant $\left(\pi, \pi^{*}\right)$ contribution, $\mathrm{T}_{2}$ is distributed on the CbDBT moiety and is principally $\left(n, \pi^{*}\right)$ in nature. Moreover, the computed SOC constant, $\xi_{\mathrm{ST}}$, is larger for $\mathrm{T}_{2}\left(4.69 \mathrm{~cm}^{-1}\right)$ than for $T_{1}\left(1.11 \mathrm{~cm}^{-1}\right)$. Therefore, theoretical results confirm that, on one side, $\mathrm{S}_{1}-\mathrm{T}_{2}$ ISC is facilitated with respect to the $S_{1}-T_{1}$ one and, on the other side, $T_{2}-T_{1} I C$ is inhibited owing to spatial separation, altogether explaining the observed anti-Kasha emission from $\mathrm{T}_{2}$. 


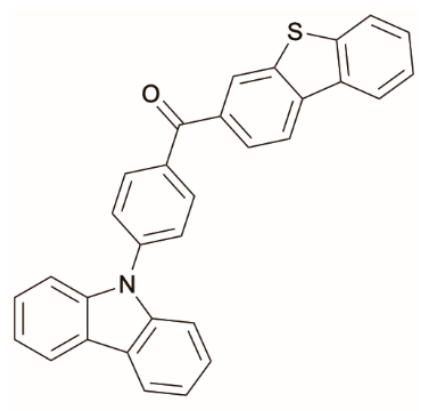

65

Scheme 21. Chemical structure of compound 65.

Wang et al. [68] prepared and fully photophysically characterized both in solution and in the solid state three 4,4'-biscarbazole derivatives, 66-68 (Scheme 22).<smiles>CCn1c2ccccc2c2cc(-c3ccc4c(c3)c3ccccc3n4-c3ccc(C(=O)c4ccccc4)cc3)ccc21</smiles>

66<smiles>CCn1c2ccccc2c2cc(-c3ccc4c(c3)c3ccccc3n4-c3ccc(C(=O)c4ccc(OC)cc4)cc3)ccc21</smiles>

67

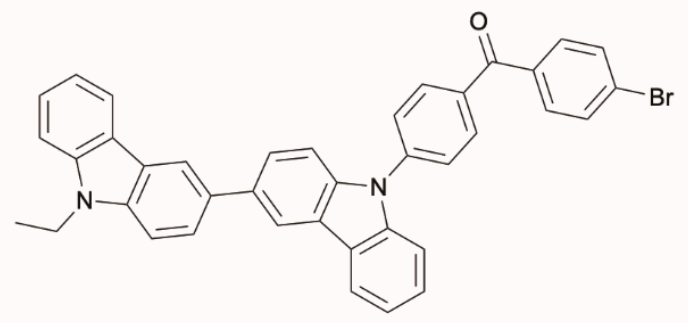

68

Scheme 22. Chemical structure of compounds 66-68.

The three compounds are not emissive (67 and 68) or are hardly emissive (66) in diluted THF or acetone solutions at RT and exhibit a fluorescence (in the 410-430 nm range) and a long-lived component (in the 460-490 nm range) at $77 \mathrm{~K}$. In solvent/nonsolvent (water) mixtures, AIE long-lived $(\approx 3 \mu \mathrm{s})$ features become visible at RT. Powders of $66-68$ at RT do not display fluorescent emissions but exhibit long-lived components with a lifetime in the microsecond range $(\Phi=35-64 \%)$ whose dual phosphorescence origin was established by steady-state and time-resolved experiment at different temperatures and assigned to $\mathrm{T}_{2}$ and $\mathrm{T}_{1}$ excited states. Assignment and mechanism of the observed dual phosphorescence were 
supported by TDDFT calculations performed at the $\omega$ B97XD/6-311G(d) level of theory. For 66, calculations revealed the presence of three triplet excited states, $\mathrm{T}_{1}$ and degenerate $\mathrm{T}_{2}$ and $\mathrm{T}_{3}$, below $\mathrm{S}_{1}$. The four excited states possess mixed $\left(n, \pi^{*}\right) /\left(\pi, \pi^{*}\right)$ character, and, being $S_{1}$ and $T_{1}$ of prevailing $\left(n, \pi^{*}\right)$ and $\left(\pi, \pi^{*}\right)$ contribution, respectively, an easy ISC from $\mathrm{S}_{1}$ to $\mathrm{T}_{1}$ can be predicted based on El-Sayed rules. Similarly, $\mathrm{T}_{2}$, despite its dominant $\left(n, \pi^{*}\right)$ character, can be easily populated from $S_{1}$ through degenerate $T_{3}$ of main $\left(\pi, \pi^{*}\right)$ nature. Radiative deactivation from $T_{2}$ competes with IC to $T_{1}$ due to the relatively large $T_{1}-T_{2}$ energy gap. Similar results were obtained for $\mathbf{6 7}$ and $\mathbf{6 8}$, for which an $S_{1}$ of predominant $\left(n, \pi^{*}\right)$ contribution and $\mathrm{T}_{1}$ and $\mathrm{T}_{2}$ dominated by a $\left(\pi, \pi^{*}\right)$ one were determined. The potentiality of the RTP features of the compounds was investigated, except for 68 having the lower quantum efficiency, in nondoped OLED devices based on their homogeneous thin films. TmPyPB, m-MTDATA (4,4',4"-Tris[(3-methylphenyl)phenylamino]triphenylamine) and TAPC were used as electron-transporting, hole-injecting and hole-transporting layers, respectively. The 67 device displays a relatively small efficiency roll-off of about $20 \%$ at $1000 \mathrm{~cd} / \mathrm{m}^{2}$ with a luminance up to $4019 \mathrm{~cd} / \mathrm{m}^{2}$. Moreover, thanks to the combination of AIE and RTP characteristics, the $\eta_{\text {ext }}$ of device $\mathbf{6 6}$ reached beyond the theoretical $\eta_{\text {ext }}$ limit of fluorescence-based OLED.

\section{Miscellaneous}

Wilson et al. [69] probed the process of exciton fission in polycrystalline thin films of pentacene, 69 (Scheme 23), by ultrafast TA spectroscopy. The authors monitored the singlet population through the detection of stimulated emissions (SEs), which were found to be excitation-dependent. The anti-Kasha blue-shifted SE obtained by excitation of higher-lying transitions was attributed to light emitted by the "hot" exciton before its relaxation to $\mathrm{S}_{1}$ (as suggested by the red arrows in Figure 17). The dynamics of triplet generation indicated that the singlet fission process does not require first relaxation to $S_{1}$ but can proceed directly from higher-lying singlet states on a similarly rapid ( $\sim 80 \mathrm{fs})$ time scale. These observations imply that pentacene $S_{1}$ state is short-lived, even on the time scale of excitonic cooling, in agreement with the very low luminescence efficiency of pentacene crystals.

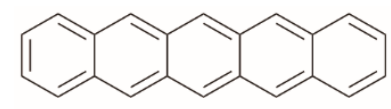

69

Scheme 23. Chemical structure of compound 69.

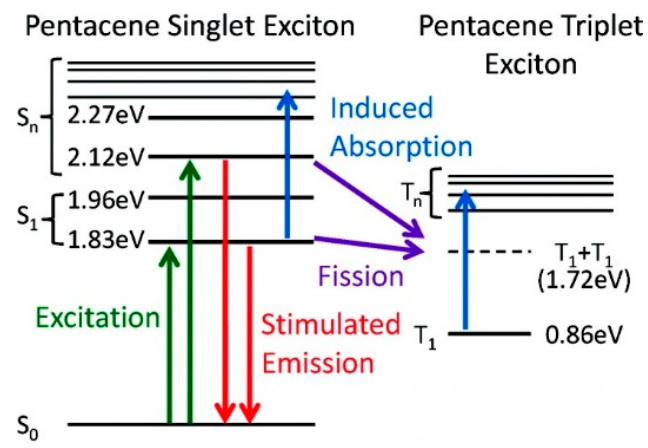

Figure 17. Energy level diagram for excitonic states in polycrystalline thin films of 69 . The labeled arrows indicate the main transitions. The energy position of the Davydov levels of the first singlet and higher-lying exciton are reported. Reprinted with permission from [69], Copyright 2011, American Chemical Society.

Some derivatives of benzaldehyde [9] were reported to emit from $T_{2}$ due to its closeness to $T_{1}$ and to the higher $S_{0}-T_{2}$ oscillator strength with respect to the $S_{0}-T_{1}$ one. However, for hydroxybenzaldehyde, 70 (Scheme 24), despite the $\mathrm{S}_{1}\left(n, \pi^{*}\right)>\mathrm{T}_{2}\left(n, \pi^{*}\right)>\mathrm{T}_{1}(\pi$, 
$\pi^{*}$ ) excited-state ordering being favorable to observing phosphorescence from $\mathrm{T}_{2}$, only phosphorescence from $\mathrm{T}_{1}$ was observed in a rigid matrix at $77 \mathrm{~K}$ [9]. In 2014, Itoh [70], speculating that anti-Kasha emission from $\mathrm{T}_{2}$ could be missed due to its weakness, performed a detailed steady-state and time-resolved investigation of $\mathbf{7 0}$ in a 1,4-dichlorobenzene matrix in the -196 to $-20{ }^{\circ} \mathrm{C}$ interval. At $77 \mathrm{~K}$ the only phosphorescent emission at $405 \mathrm{~nm}$ was assigned to the $\mathrm{T}_{1}\left(\pi, \pi^{*}\right)$ state. At $-70{ }^{\circ} \mathrm{C}$ an additional phosphorescent contribution at $391 \mathrm{~nm}$ of $\mathrm{T}_{2}\left(n, \pi^{*}\right)$ origin, with intensity increasing with temperature up to $-20^{\circ} \mathrm{C}$, was observed. A $\Delta \mathrm{E}\left(\mathrm{T}_{1}-\mathrm{T}_{2}\right)$ equal to $0.11 \mathrm{eV}$ was estimated from both steady-state and timeresolved experiments which allowed the drawing of the mechanism reported in Figure 18 in which the thermal population of $\mathrm{T}_{2}$ from $\mathrm{T}_{1}$ justifies the observed emissive behavior.<smiles>O=Cc1ccc(O)cc1</smiles>

Scheme 24. Chemical structure of compound 70.

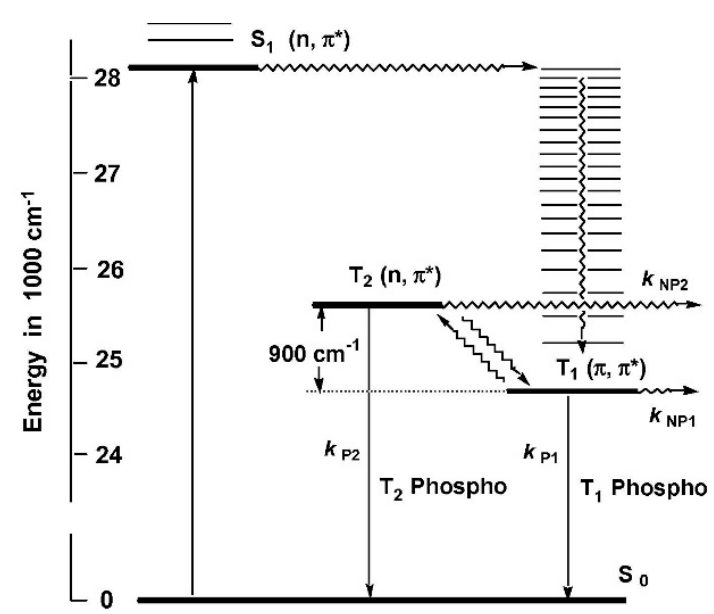

Figure 18. Energy level scheme showing the relaxation processes for $\mathbf{7 0}$ in a $p$-dichlorobenzene matrix: $\mathrm{k}_{\mathrm{P} 1}$ and $\mathrm{k}_{\mathrm{NP} 1}$ are, respectively, the radiative and nonradiative rate constants of $\mathrm{T}_{1} ; \mathrm{k}_{\mathrm{P} 2}$ and $k_{\mathrm{NP2}}$ are those of $\mathrm{T}_{2}$. Reprinted from [70], Copyright 2014, with permission from Elsevier.

While pyrene, previously reported as an exception to Kasha rule [9], was recently demonstrated [14] to emit uniquely from $S_{1}$, the introduction of dicyano methane substituents on the pyrene scaffold to generate compounds $\mathbf{7 1}$ and $\mathbf{7 2}$ (Scheme 25) promotes, in DCM solution, an intense ICT emission that originates from $S_{2}$, while no radiative deactivation is obtained upon $S_{0}-S_{1}$ excitation [71] (see Figure 19). Violation of the Kasha rule was attributed to the large $S_{1}-S_{2}$ energy gap $(0.98$ and $0.94 \mathrm{eV}$ in $\mathbf{7 1}$ and $\mathbf{7 2}$, respectively, as determined by DFT and TDDFT calculations at B3LYP/6-31G $(\mathrm{d}, \mathrm{p})$ level) favoring emission from $S_{2}$ over $S_{2}-S_{1}$ IC as a consequence of the reduced overlap between vibrational levels of $S_{1}$ and $S_{2}$. 
<smiles>CC(C)(C)c1cc2c(C(C)(C)C)cc3ccc4cc(C(C)(C)C)cc5c(=C(C#N)C#N)c(=C(C#N)C#N)c(c1)c2c5c43</smiles>

71<smiles></smiles>

72

Scheme 25. Chemical structure of compounds 71 and $\mathbf{7 2 .}$
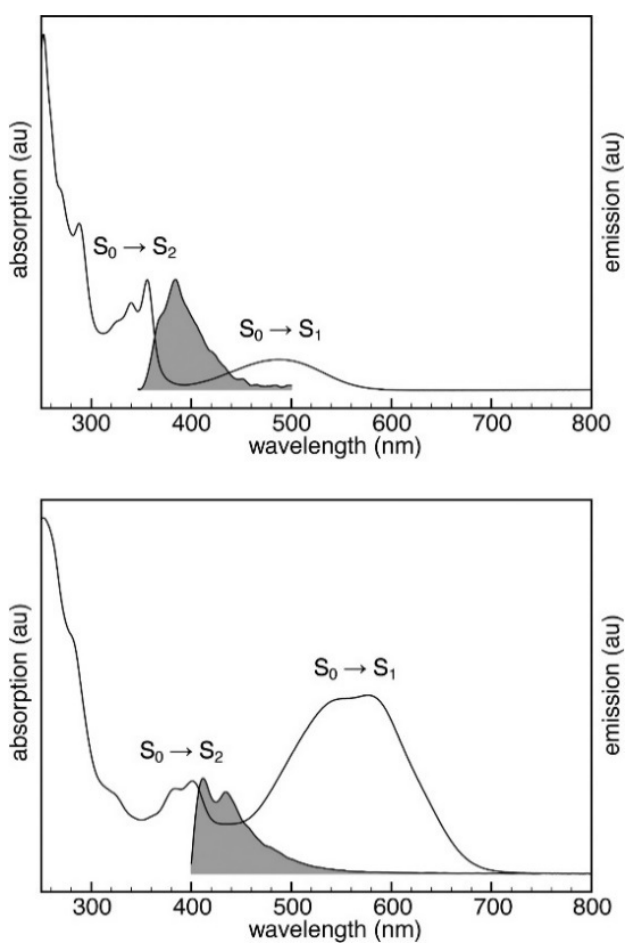

Figure 19. Absorption (white) and emission (gray) features for 71 (top) and 72 (bottom) in DCM. Reprinted with permission from [71], Copyright 2014, American Chemical Society.

Bis(bora)calix[4]arene, 73 (Scheme 26), was reported by Arimori et al. [72] as a fluorescent sensor selective for fluoride due to a decrease in UV absorption and fluorescence upon $\mathrm{F}^{-}$binding. In 2016, Jin et al. [73] performed a thorough DFT/TDDFT study to interpret this behavior. Calculations were performed by using a GGA functional, PBE1W, which provided a better agreement with experimental data, and the 6-311G(d) basis set. The prompt fluorescence of 73 was assigned to emission from $S_{2}$ owing to a sizable $\Delta E\left(S_{1}-S_{2}\right)$ gap $(0.48 \mathrm{eV})$ and very low $\mathrm{S}_{0}-\mathrm{S}_{1}$ oscillator strength. The quenching of fluorescence observed for the fluoride-binding complex 73-F was explained by a theoretically predicted fast IC to a dark $S_{1}$ state. 


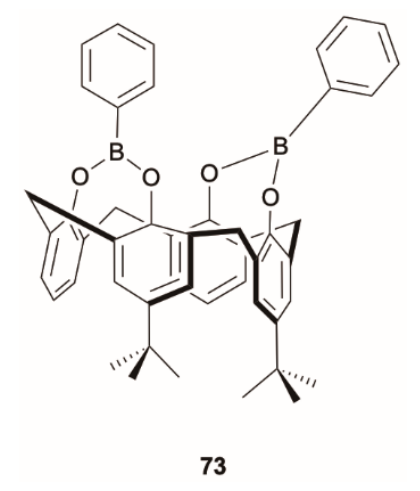

Scheme 26. Chemical structure of compound 73.

Qian et al. [74] reported on a series of boron difluorohydrazone derivatives, 7481 (Scheme 27), showing enhanced emission in the solid state and in highly viscous environments and a quenched emission in nonviscous solvents. Based on DFT/TDDFT calculations at the PBE/TZVP level, the authors attributed this behavior to the presence, in these molecular rotors, of an $S_{1}$ dark state and of a higher excited state, $S_{n}$ (with $n>1$, varying within the series of compounds), having high oscillator strength. In nonviscous solvents, the free intramolecular rotation in the excited state leads to a conformation having a low $S_{n}-S_{1}$ energy gap, which allows rapid $S_{n}-S_{1}$ IC and then very weak emission or nonradiative decay to $S_{0}$. Highly viscous solvents, on the other hand, inhibit the formation of the nonradiative excited-state structure, decreasing the rate of $S_{n}-S_{1}$ IC and then resulting in anti-Kasha emission from $S_{n}$. Such behavior was supported by a strict correlation between the viscosity sensitivity of the fluorescence and the computed barrier to rotation in the excited state: the higher the barrier, the greater the viscosity sensitivity. Moreover, the anti-Kasha emission was experimentally confirmed in $\mathbf{8 1}$ by the observation of different absorbance and circular dichroism band shapes at $77 \mathrm{~K}$, a feature that can be explained by the existence of two or more distinct electronic transitions. Furthermore, a weak emission emerged when excitation was shifted towards lower energies, indicating that the molecule is relaxing from the dark state $S_{1}$.
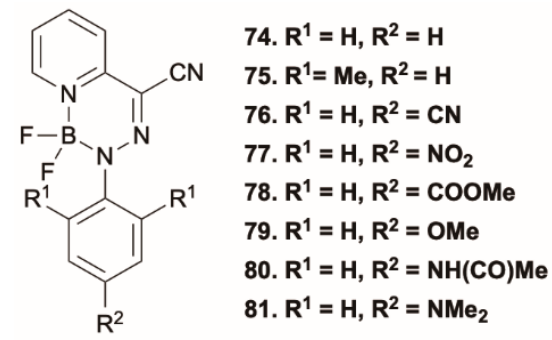

Scheme 27. Chemical structure of compounds 74-81.

Subsequently, Zhou et al. [75], contesting the use of the pure functional PBE adopted by Qian et al. [74] for 74-81, performed a new TDDFT study on the same series of compounds using the B3LYP and CAM-B3LYP functionals, besides complete active space self-consistent field (CASSCF) calculations. It was reported that $S_{1}$ for the same series of compounds 74-81 is not a dark but a bright state, which calls into question the anti-Kasha origin of the enhanced emission of these compounds in viscous environments. The authors proposed an alternative origin, based on restriction, in viscous solvents, of flip-flop motion promoting fast $S_{1}-S_{0}$ IC. Confutation of the previously proposed anti-Kasha behavior of these compounds, however, still deserves experimental validation.

Zhou et al. [76] reported single-molecule white light emission from dibenzo[ $a, c]$ phenazine, 82 (Scheme 28). In solution, 82 displays steady-state PL dominated by a structured phosphorescence with a lifetime of $2.7 \mu \mathrm{s}$, accompanied by a fluorescent emission. This latter was associated to an $S_{1}$ state with $\left(n, \pi^{*}\right)$ character while the phosphorescence was 
assigned to a $\left(\pi, \pi^{*}\right) \mathrm{T}_{1}$ level, with $\mathrm{S}_{1}-\mathrm{T}_{1}$ ISC facilitated by El-Sayed considerations (see Figure 20), as confirmed by the very short fluorescence lifetime of $82(0.07 \mathrm{~ns})$ [77]. Powders of 82 display multiple emissions comprising one $S_{1}-S_{0}$ fluorescence and two RTPs, reaching Commission Internationale de l'Éclairage (CIE) coordinates of $(0.28,0.33)$ and $\Phi$ of $1 \%$. From steady-state measurements at variable temperature and time-resolved studies, the authors demonstrate that the two RTPs are relatively independent of each other and excluded a $\mathrm{T}_{1}-\mathrm{T}_{\mathrm{n}}$ thermally activated reversed IC. With the support of TDDFT calculations, performed at the B3LYP/6-31G $(\mathrm{d}, \mathrm{p})$ level of theory, the high-energy RTP was assigned to a $\mathrm{T}_{2}$ state of $\left(n, \pi^{*}\right)$ character. $\mathrm{T}_{2}-\mathrm{T}_{1} \mathrm{IC}$ competes with $\mathrm{T}_{2}$ radiative deactivation, leading to the dual phosphorescent emission. The excitation wavelength dependency of the RTPs demonstrated that the high-energy one could be efficiently generated through multiple alternative ISC channels $\left(S_{2}-T_{2}\right.$ and $S_{1}-T_{2}$, see Figure $\left.20 \mathrm{c}\right)$.<smiles>c1ccc2c3nc4ccccc4c4ccccc4c-3nc2c1</smiles>

82

Scheme 28. Chemical structure of compound $\mathbf{8 2 .}$

(a)

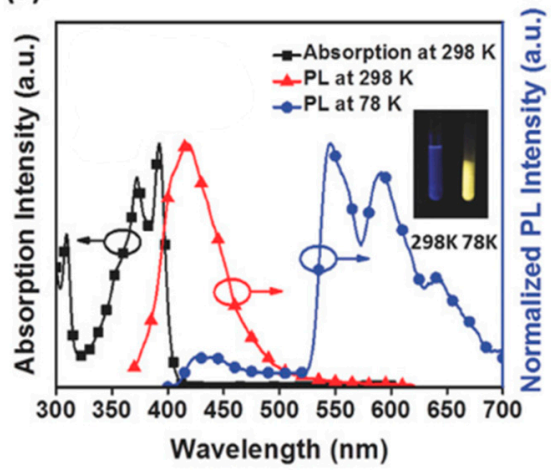

(b)

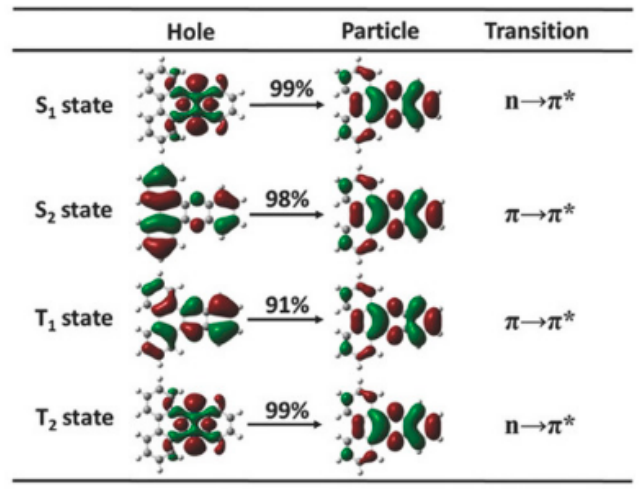

Figure 20. Cont. 
(c)

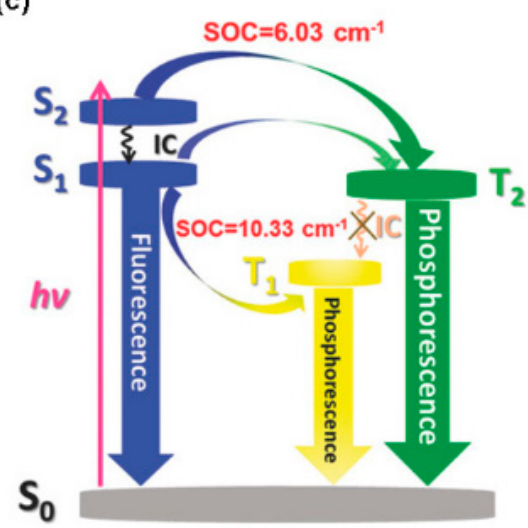

Figure 20. (a) Absorption and emission spectra of 82 in THF solution at 298 and $78 \mathrm{~K}$. The insets are the photos of the steady-state emission. The excitation wavelength is $390 \mathrm{~nm}$. (b) Natural transition orbital (NTO) images of the $\mathrm{S}_{1}, \mathrm{~S}_{2}, \mathrm{~T}_{1}$ and $\mathrm{T}_{2}$ excited states of 82. The NTO images of each excited state represent their transition forms from the "hole" unity to the "particle" unity, respectively, and the percentages on the arrows are the proportions of the transitions. (c) Schematic Jablonski diagram with SOC matrix elements of RTP-related ISC processes in 82. Adapted with permission from [76].

Peng et al. [78] investigated the photophysical properties in different states of a series of amino benzothiadiazoles. Anti-Kasha dual emission was observed in solution for 83-87 (Scheme 29) and was correlated to the strength of the electronic communication between the amino group and the benzothiadiazole moiety. In fact, by increasing the CT character of the molecule, the molecular rigidity is increased and $\mathrm{S}_{2}-\mathrm{S}_{1} \mathrm{IC}$ is inhibited. For 83 and 84, having the strongest $\mathrm{CT}$ character and the highest planarity among the members of the family, excitation-dependent dual emission in the visible region was observed both in toluene and acetonitrile solutions. Compounds 85 and 86, characterized by reduced electron-donating ability from the amino group, display dual emission only in acetonitrile where the ICT is enhanced. For 87, dual emission appears in both solvents but is hardly visible in acetonitrile.<smiles>[R1]Nc1ccc(-c2ccc(OC)cc2)c2nsnc12</smiles>

83. $R^{1}=H, R^{2}=n B u$

84. $R^{1}=H, R^{2}=$ adamantyle

85. $R^{1}=H, R^{2}=P h$

86. $R^{1}=H, R^{2}=4-M e O-P h$

87. $\mathbf{R}^{1}=4-\mathrm{MeO}-\mathrm{Ph}, \mathbf{R}^{2}=4-\mathrm{MeO}-\mathrm{Ph}$

Scheme 29. Chemical structure of compounds 83-87.

Sun et al. [79] and Liu et al. [80] revealed a family of highly twisted $o, o^{\prime}$-substituted binaphthyls, 88-92, featuring a D- $\pi-\mathrm{A}$ structure with ICT character within each aryl group (Figure 21). These compounds, unlike the "normal" D- $\pi-\mathrm{A}$ molecules, consist of two independent $D-\pi-A$ subunits, each of them bearing either dimesitylboryl, $\operatorname{Mes}_{2} B(88,89,92)$ or $\mathrm{CHO}(90)$ or $\mathrm{CN}(\mathbf{9 1})$ groups as strong electron acceptors, and $\mathrm{NR}_{2}$ groups, with $\mathrm{R}=\mathrm{Me}$ $(88,90-92)$ or $\mathrm{Ph}(\mathbf{8 9})$ as donors. All these compounds revealed temperature-dependent dual fluorescence and switchable circularly polarized luminescence (CPL). Comparison between 88 and 89 [79] indicates that, in the presence of the less bulky $\mathrm{NMe}_{2}$ group (88), the two fluorescence bands are well separated in polar solvents and are very sensitive to temperature, enabling the use of $\mathbf{8 8}$ as a ratiometric fluorescence thermometer. On the other hand, for 89, the dual emission was observed only in the CPL spectra, probably due to the overlap of the two fluorescences. Combining experimental and theoretical results, the latter obtained by DFT and TDDFT calculations at the PBE0/6-31G(d) level 
of theory, the dual fluorescence of $\mathbf{8 8}$ was assigned to deactivation from $S_{1}$ and $S_{2}$. The computed excitation energies of these two states were very similar $(\Delta \mathrm{E}=0.07 \mathrm{eV})$, justifying a thermal equilibrium population between them which explains the observed temperaturedependent dual fluorescence. Moreover, the optimized geometries of $S_{1}$ and $S_{2}$ reveal different features, $S_{1}$ having mainly inter-subunit $C T$ character and $S_{2}$ having mixed intrasubunit $\mathrm{CT}$ and LE character. Calculations on $\mathbf{8 9}$ provided essentially identical features; owing to its higher structural rigidity with respect to $\mathbf{8 8}$, only small changes are observed in the optimized $S_{1}$ and $S_{2}$ geometries with respect to the ground state. This explains the observed overlap of the $S_{1}-S_{0}$ and $S_{2}-S_{0}$ deactivations.

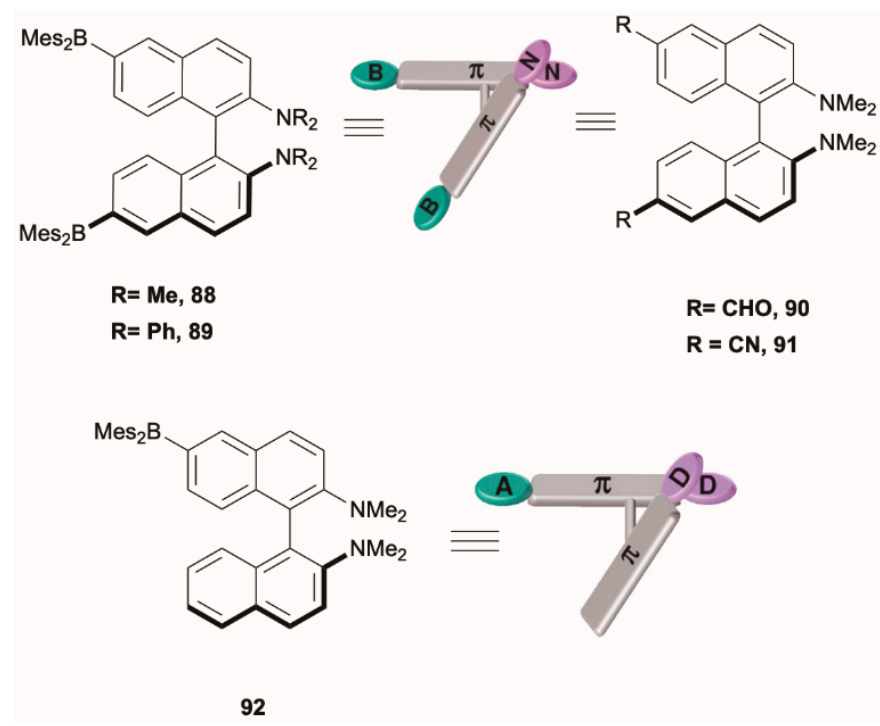

Figure 21. Schematic presentation and structure of $o, o^{\prime}$-binaphthyls 88-92 consisting of two D- $\pi-\mathrm{A}$ subunits. Adapted with permission from [80].

From comparative analysis of the effect of the A group in $\mathbf{8 8 ,} 90$ and 91 [80], it appeared that the acceptor groups greatly affect the relative intensity and the energy separation of the two emissions. The reference compound 92, lacking one electron-accepting group, was found to possess less sensitive temperature-dependent dual fluorescence, indicating that the symmetric structure of $\mathbf{8 8 - 9 1}$ is very important for realizing highly sensitive temperature-dependent dual fluorescence.

Liu et al. [81] reported a study on intramolecular $\mathrm{O}-\mathrm{H} \cdots \mathrm{S}$ H-bond formation in a thione derivative which displays dual RTP due to a remarkable photoinduced intramolecular H-bond on/off switching reactions. The corresponding methylated compound, 93 (Scheme 30), which lacks the dual RTP, represents instead an example of anti-Kasha fluorescence (see Figure 22). In cyclohexane at RT, 93 displays dual emission comprising one fluorescence (at $380 \mathrm{~nm}$ ) and an RTP (at $690 \mathrm{~nm}$ ). Through a deep photophysical analysis, integrated with DFT and TDDFT calculations at the B3LYP/6-311++G(3df,3pd) level of theory, the authors assigned the first excited state, $S_{1}$, to an optically forbidden $\left(n, \pi^{*}\right)$ transition and the higher-lying $S_{2}$ state to an allowed $\left(\pi, \pi^{*}\right)$ one. The calculated $\Delta E\left(S_{2}-S_{1}\right)$ energy gap, amounting to about $1.37 \mathrm{eV}$, justifies an anomalously slow $\mathrm{S}_{2}-\mathrm{S}_{1} \mathrm{IC}$, allowing the radiative $S_{2}-S_{0}$ transition to become competitive.

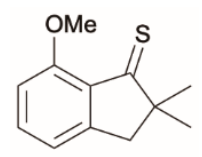

93

Scheme 30. Chemical structure of compound 93. 


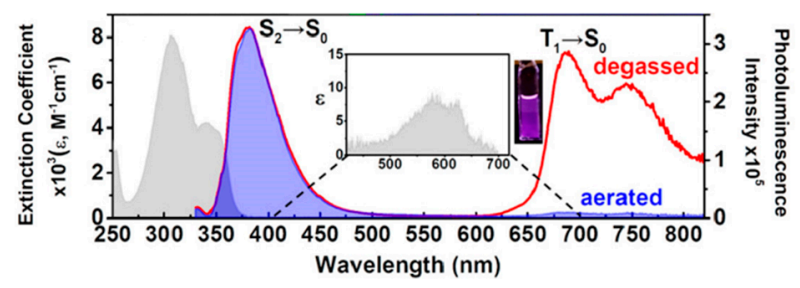

Figure 22. Absorption (gray filled area) and emission spectra (blue filled area and solid red line) of 93 in cyclohexane at room temperature. Insets: expansions of the absorption spectra over specified wavelength regions. $\lambda_{\mathrm{ex}}: 350 \mathrm{~nm}$. Note that the labeling "aerated" refers to aeration in atmosphere. Adapted with permission from [81], Copyright 2019, American Chemical Society.

Guo et al. [82] reported three donor-acceptor (D-A) compounds, 94-96 (Scheme 31), consisting of electron-donating phenoxazine and fluorene derivatives coupled with an electron-withdrawing benzoyl core, that are weakly emissive in solution but display DF upon aggregation. DFT/TDDFT calculations using the PBE0 functional were employed to rationalize the emission mechanism of $\mathbf{9 4}$ as the representative of the family. The obtained oscillator strength for $S_{1}$ was almost zero $(f=0.0006)$, indicating that $S_{1}$ is nonemissive. On the other hand, the optimization of the $S_{2}$ geometry provided a relatively large $f$ value (0.0523), suggesting that the efficient emission of $\mathbf{9 4}$ corresponds to an anti-Kasha radiative deactivation from $\mathrm{S}_{2}$.<smiles>CC1(C)c2ccccc2-c2ccc(C(=O)c3ccc(N4c5ccccc5Oc5ccccc54)cc3)cc21</smiles>

94<smiles>O=C(c1ccc(N2c3ccccc3Oc3ccccc32)cc1)c1ccc2c(c1)C(c1ccccc1)(c1ccccc1)c1ccccc1-2</smiles>

95<smiles>O=C(c1ccc(N2c3ccccc3Oc3ccccc32)cc1)c1ccc2c(c1)C1(c3ccccc3-2)c2ccccc21</smiles>

Scheme 31. Chemical structure of compounds 94-96.

Additionally, natural transition orbital (NTO) analysis, both in THF and in solid state, has been performed. For $\mathrm{S}_{2}$, a typical CT state was predicted, while a significant LE feature was found for $\mathrm{T}_{3}$. Moreover, the small $(0.22 \mathrm{eV}) \mathrm{S}_{2}-\mathrm{T}_{3}$ energy gap calculated in solid state makes an rISC process highly favorable; thus, in the solid state, $T_{3}$ can be converted to $S_{2}$, supporting the subsequent anti-Kasha delayed emission (see Figure 23). The full emissive 
mechanism in the solid state can be therefore explained by a reduced IC and a promoted ISC, with respect to the solution, resulting in the DF.
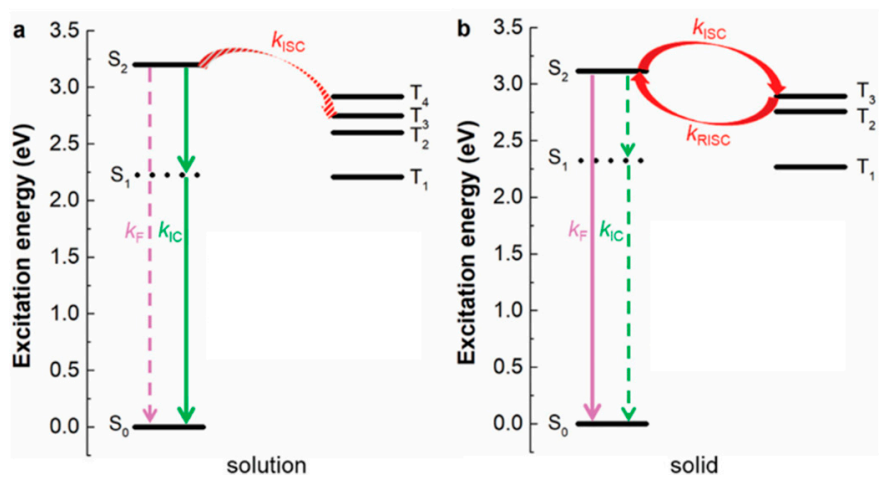

Figure 23. Adiabatic excitation energies for 94 (a) in THF solution and (b) in solid state. Adapted with permission from [82].

Such aggregation-induced delayed fluorescence (AIDF) appears particularly useful for OLED applications since the use of fluorescent emission, with respect to phosphorescence, allows the problem of efficiency roll-off to be reduced, while the AIE property of the compounds allows the fabrication of efficient nondoped devices. Therefore, the EL behavior of the compounds was investigated in nondoped and doped OLEDs, and external quantum efficiency, $\eta_{\text {ext }}$, of up to $14.3 \%$ was obtained in the device of 95 . At $1000 \mathrm{~cd} \mathrm{~m}^{-2}$ the device kept excellent $\eta_{\text {ext }}$ of $14.1 \%$, and the external quantum efficiency roll-off was as small as $1.4 \%$, indicative of the superior efficiency stability of the device based on these AIDF materials.

Shi et al. [83] proposed a strategy to obtain ratiometric sensing for quantitative analysis of biomolecules in a dynamic cellular environment by using dyes able to switch from a close Kasha form to an open anti-Kasha one characterized by dual emission. In particular, 97 (Scheme 32), where fluorescein is covalently linked to a chromene unit, displays in solution two fluorescences at 520 and $700 \mathrm{~nm}$, assigned, through DFT-TDDFT studies at M06-2X/Def2-SVP level, to emissions from $S_{2}$ and $S_{1}$, respectively. According to the quantum chemical calculations, a $\Delta \mathrm{E}\left(\mathrm{S}_{2}-\mathrm{S}_{1}\right)$ equal to $0.74 \mathrm{eV}$ was determined. Such a high energy gap, together with the different $S_{2}$ and $S_{1}$ orbital localization, the former mainly on the fluorescein fragment and the latter on the chromene moiety, justify the slow $S_{2}-S_{1}$ IC resulting in the observed dual fluorescence. For the corresponding closed form of $\mathbf{9 7}$, only Kasha emission from $S_{1}$ was recorded (see Figure 24).

Starting from 97, similar compounds $(\mathbf{9 8}, 99)$ (Scheme 32) were prepared and studied. Through D- $\pi-\mathrm{A}$ structural modifications, the $\Delta \mathrm{E}\left(\mathrm{S}_{2}-\mathrm{S}_{1}\right)$ gap can be modulated to provide diverse anti-Kasha/Kasha chromophores exhibiting an invariant $S_{1}-S_{0}$ NIR Kasha emission and an anti-Kasha visible $\mathrm{S}_{2}-\mathrm{S}_{0}$ one. In the physiological $\mathrm{pH}$ range, these chromophores remain in their open form, emitting from both $S_{2}$ and $S_{1}$. A quantitative sensing platform can be realized by replacing the hydroxy group with different biorecognition units (Figure 25) When the probes in their closed form (with only the NIR emission from $\mathrm{S}_{1}$ ) encounter the corresponding analyte, the hydroxyl group is restored, turning the probes to their open form at the desirable physiological $\mathrm{pH}$, with both $\mathrm{S}_{2}$ and $\mathrm{S}_{1}$ emissions. The invariant NIR Kasha emission from the $S_{1}$ state acts as internal reference while the green emission from the anti-Kasha-active $S_{2}$ state linearly increases with the concentration of targeted analyte. Using this strategy, the authors demonstrated the ratiometric quantification of cysteine and glutathione in living cells and animals. 
<smiles>N#CC(C#N)=C1C=C(C=Cc2c3oc4ccc5c(-c6ccccc6C(=O)O)c6ccc(=O)cc6oc5c4cc4c(c23)CCCC4)Oc2ccccc21</smiles>

97<smiles></smiles>

98<smiles></smiles>

99

Scheme 32. Chemical structure of compounds 97-99.
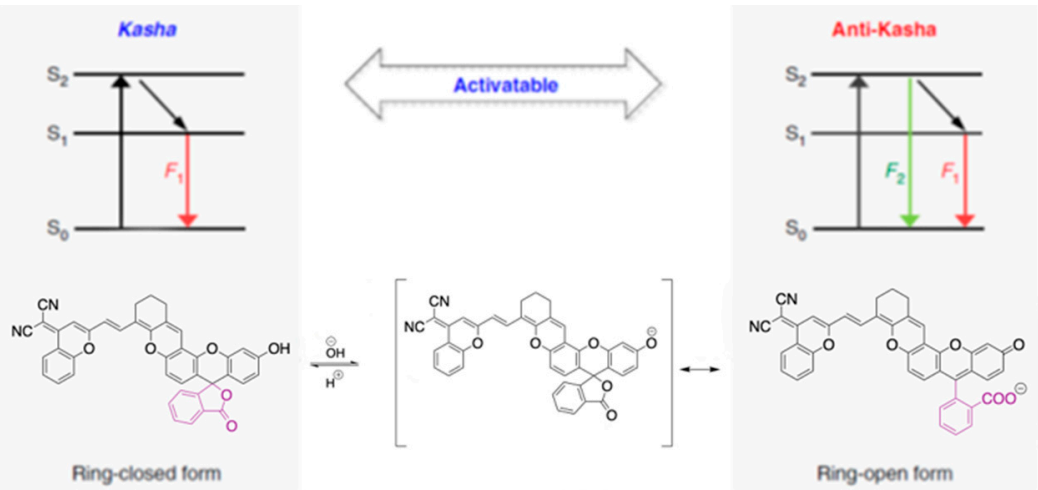

Figure 24. Schematic illustration of spirolactone switch-controlled $\mathbf{9 7}$ for tuning anti-Kasha/Kasha properties. Reprinted from [83]. 


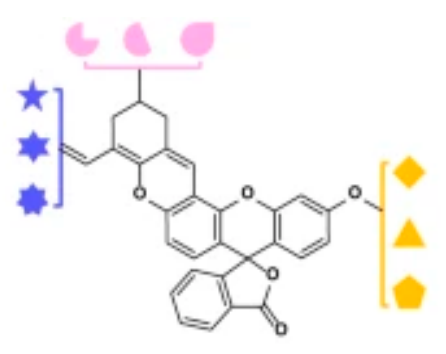

Ring-closed form IFC Probes

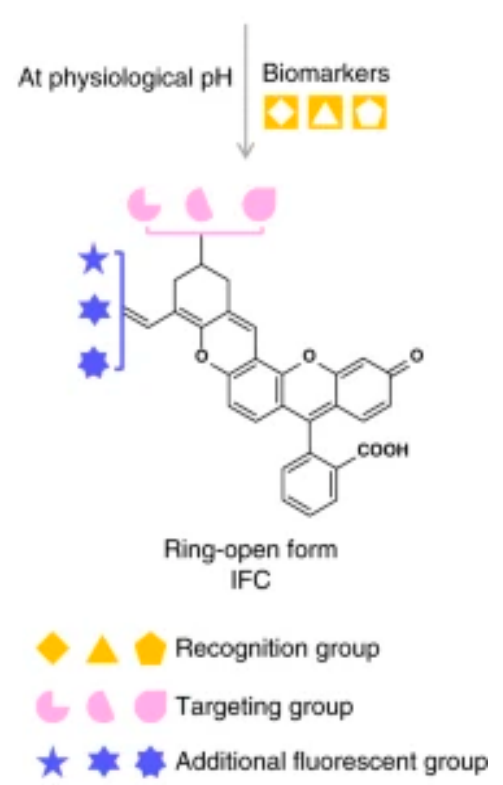

Figure 25. Chemical structures of 97-99 with different biomolecular-recognition groups as quantitative sensing platform (IFC stands for integrated fluorescein with chromene). Reprinted/adapted from [83].

Luo et al. [84] reported dual TADF emission in a single luminophore arising from anti-Kasha/Kasha pathways from different excited electronic states (see Figure 26). The authors designed the unsymmetrical D-A-D' molecular structure 100 (Scheme 33) (where $\mathrm{D}=$ phenothiazine, $\mathrm{D}^{\prime}=\mathrm{N}-(1 \mathrm{H}$-indole-5-yl) acetamide and $\mathrm{A}=$ diphenylsulfone $)$ which possesses two CT states localized on the $\mathrm{D}-\mathrm{A}$ and $\mathrm{D}^{\prime}-\mathrm{A}$ subunits of the molecule, as elucidated by means of B3LYP/6-31G(d) DFT and TDDFT calculations. For each CT singlettriplet pair, the calculated energy splitting was found to be less than $0.1 \mathrm{eV}$, allowing, both in diluted solution and in the aggregated state (THF/water, solvent/nonsolvent mixture), very fast ISC and rISC rates that produce two independent DFs with different colors and lifetimes. Due to its favorable features, $\mathbf{1 0 0}$ was successfully employed in timeresolved fluorescence cell imaging. The main advantage of this probe was its capability to provide complementary dual-channel lifetime mapping that reduces time-resolved imaging distortions.

Wu et al. [85] presented three heterocyclic 1,2-diphenyl ethylene derivatives, 101-103 (Scheme 34), that, at ambient conditions in the solid state, display excitation wavelengthdependent multiple emission, comprising prompt, delayed and RTP components originating from first and higher-energy excited singlet and triplet levels. The first and upper excited singlet and triplet states were responsible for the observed dual fluorescence and dual phosphorescence, as supported by QM/MM calculations with TDDFT excitation energies for the QM region computed at B3LYP/6-31G(d) level. In particular, prompt fluorescence from $S_{1}$ and prompt and TADF emission from $S_{2}$ were observed for all com- 
pounds, while dual $\mathrm{T}_{2} / \mathrm{T}_{1}$ RTP was recorded only for 101, 102 and 103 displaying single RTP from $\mathrm{T}_{2}$ (see Figure 27).

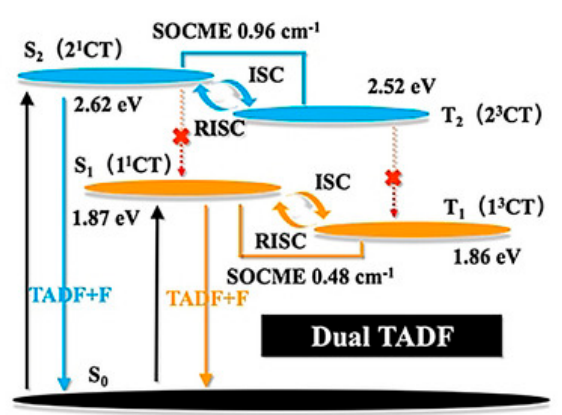

Figure 26. Possible mechanism for the dual TADF proposed for $\mathbf{1 0 0}$ on the basis of TDDFT calculations (SOCME represents spin-orbit coupling matrix element). Adapted with permission from [84].

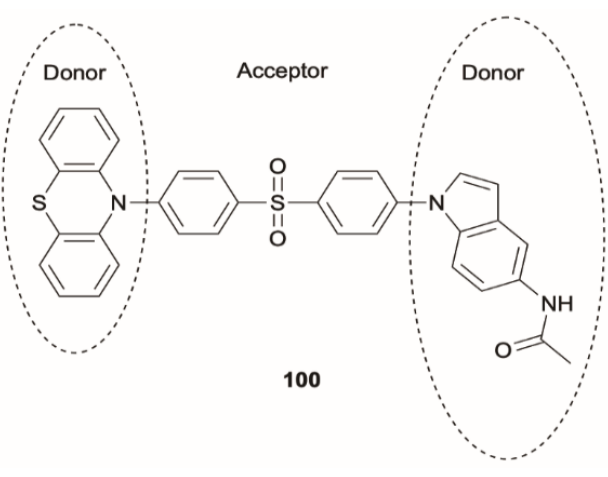

Scheme 33. Chemical structure of compound 100.

For 101, an $S_{2}$ level of mainly $\left(\pi, \pi^{*}\right)$ character, energetically close to triplet states of mixed $\left(n, \pi^{*}\right) /\left(\pi, \pi^{*}\right)$ nature, and an $S_{1}$ and its close triplets of $\left(\pi, \pi^{*}\right)$ symmetry were calculated. For the other two compounds, an $\left(n, \pi^{*}\right) S_{2}$ level close to $\left(\pi, \pi^{*}\right)$ triplets together with an $S_{1}$ and its adjacent triplets of $\left(\pi, \pi^{*}\right)$ symmetry were computed. Therefore, based on symmetry considerations and the small $\mathrm{E}\left(\mathrm{S}_{2}-\mathrm{T}_{\mathrm{n}}\right)(0.08,0.04$ and $0.02 \mathrm{eV}$ for 101, 102 and 103, respectively), an easy DF from $S_{2}$ and a negligible one from $S_{1}$ were predicted. Moreover, the large $\Delta \mathrm{E}\left(\mathrm{T}_{2}-\mathrm{T}_{1}\right)$ of $\mathbf{1 0 1}, 1.40 \mathrm{eV}$, explains its observed dual RTP.<smiles>O=C1OC(=C2OC(=O)c3ccccc32)c2ccccc21</smiles>

101<smiles>O=C1OC(=C2SC(=O)c3ccccc32)c2ccccc21</smiles>

102<smiles>O=C1SC(=C2SC(=O)c3ccccc32)c2ccccc21</smiles>

103

Scheme 34. Chemical structure of compounds 101-103.

Sun et al. [86] reported a tetraphenylethene-substituted Schiff base, 104 (Scheme 35), which exhibits water-induced fluorescent switch from weak yellow-green Kasha emission to intense sky blue anti-Kasha one. According to TDDFT calculations at the B3LYP/6$31 \mathrm{G}(\mathrm{d})$ level of theory, in nonprotic solvents, an ESIPT process involving an intramolecular $\mathrm{O}-\mathrm{H} \cdots \mathrm{N}$ hydrogen bond is expected to lead to a ketone structure, responsible for the Kasha yellow emission (see Figure 28). When water was added, an intramolecular hydrogen bond was broken in favor of an intermolecular one with a water molecule. ESIPT associated with 
this interaction leads to a solvated ketone structure whose $440 \mathrm{~nm}$ emission was assigned to radiative deactivation from $S_{2}$, and the $S_{2}-S_{1} I C$ was inhibited by the large $\Delta E\left(S_{2}-S_{1}\right)$, $0.54 \mathrm{eV}$. It should be underlined that this ESIPT process is correctly included in the present review, despite what was mentioned in the introduction, since it results in the formation of a new species from which anti-Kasha emission is observed. A practical use of $\mathbf{1 0 4}$ in rewritable papers and fluorescent sensors was developed with potential applications in information security protection, multilevel anticounterfeiting and data storage.

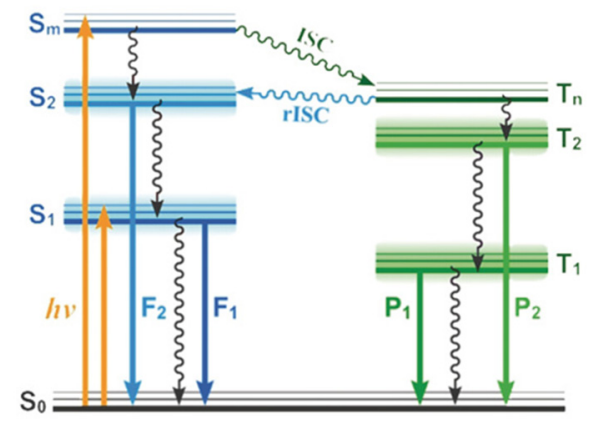

Figure 27. Suggested mechanism for the upper excited state fluorescence from neat solid 101, 102 and 103, based on DFT/TDDFT calculations. Adapted with permission from [85].<smiles>N#Cc1ccc(O)c(/C=N/c2ccc(C(=C(c3ccccc3)c3ccccc3)c3ccccc3)cc2)c1</smiles>

104

Scheme 35. Chemical structure of compound 104.

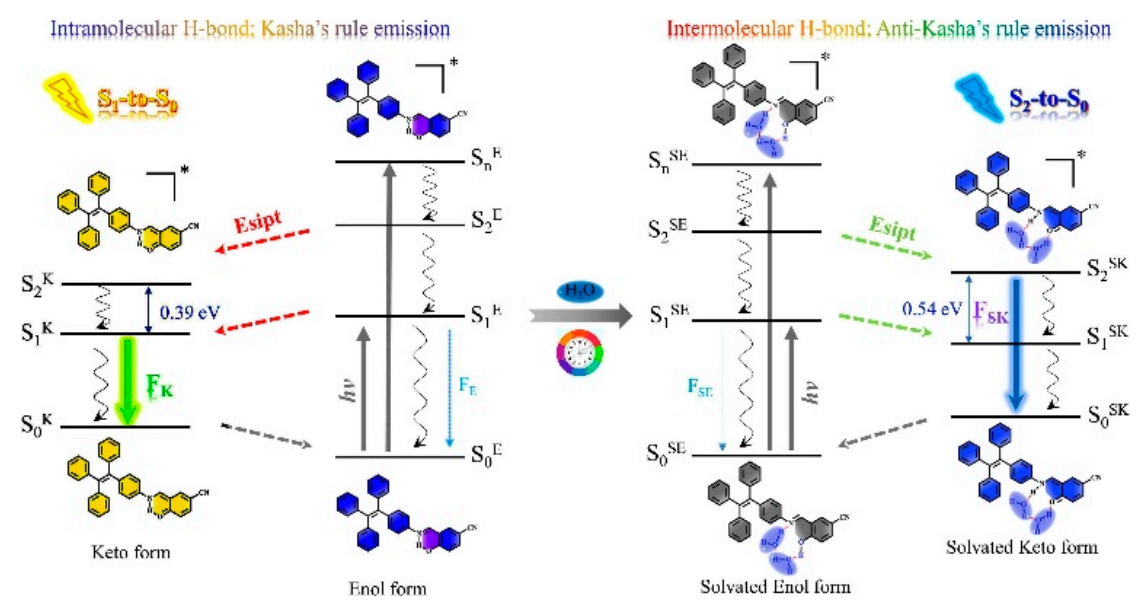

Figure 28. Possible mechanism for the dynamic stimuli-responsive fluorescence switch. $\mathrm{S}_{0}{ }^{\mathrm{E}}$ : ground state of enol form of 104; $\mathrm{S}_{\mathrm{n}}{ }^{\mathrm{E}}$ : excited state of enol form of $104 ; \mathrm{S}_{0}{ }^{\mathrm{K}}$ : ground state of keto form of 104; $\mathrm{S}_{\mathrm{n}}{ }^{\mathrm{K}}$ : excited state of keto form of 104; $\mathrm{S}_{0}{ }^{\mathrm{SE}}$ : ground state of enol form of solvated 104; $\mathrm{S}_{\mathrm{n}}{ }^{\mathrm{SE}}$ : excited state of enol form of solvated 104; $\mathrm{S}_{0} \mathrm{SK}$ : ground state of keto form of solvated $104 ; \mathrm{S}_{\mathrm{n}}{ }^{\mathrm{SK}}$ : excited state of keto form of solvated 104. Adapted from [86], Copyright 2021, with permission from Elsevier.

Due to the presence of $\left(\pi, \pi^{*}\right)$ and $\left(n, \pi^{*}\right)$ levels, coumarin exhibits efficient El-Sayedallowed ISC and therefore represents a useful scaffold to populate triplet levels in the 
absence of heavy atoms [87]. Jhun et al. [87] investigated a series of D-coumarin derivatives where $\mathrm{D}$ represents units with different oxidation potentials. Among the studied compounds, 10H-spiro(acridine-9,9'-fluorene), 105; 9,9'-dimethyl-9,10-dihydroacridine, 106; and 10H-phenoxazine, 107 (Scheme 36), display anti-Kasha dual emission in toluene solution comprising a high-energy fluorescence at $420 \mathrm{~nm}$ of $\left(\pi, \pi^{*}\right)$ with partial CT character and a low-energy one in the 470-580 $\mathrm{nm}$ region of ICT character. Steady-state and time-resolved results revealed the TADF nature of the low-energy emission, which involves thermal repopulation of the ${ }^{1} \mathrm{ICT}$ state from a $\operatorname{close}^{3}\left(n, \pi^{*}\right)$ one. This latter is populated through El-Sayed-allowed ISC from the ${ }^{1}\left(\pi, \pi^{*}\right)$ level since both states are localized on the coumarin moiety (see Figure 29). Intriguingly, such dual fluorescence is produced from $\left(\pi, \pi^{*}\right)$ and ICT transitions, both usually characterized by high oscillator strength. On the contrary, a single Kasha fluorescence was observed for the reference carbazole-coumarin compound, where the low $\left(\pi, \pi^{*}\right)$-ICT energy gap $(0.23 \mathrm{eV})$, smaller than those of the other investigated D-coumarin derivatives, allows a faster IC to the ${ }^{1}$ ICT state rather than ISC to the ${ }^{3}\left(n, \pi^{*}\right)$ one. It has to be noted that TDDFT calculations on this series of compounds, aimed at investigating the nature of the emissive state(s), revealed that the B3LYP functional provided results closer to the experimental values with respect to other tested (CAM-B3LYP and PBE0) functionals.

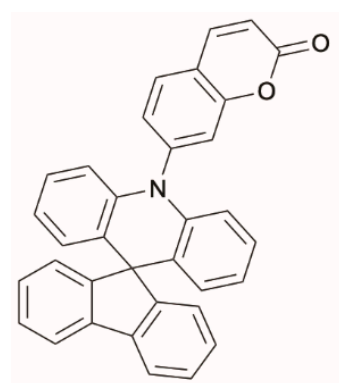

105

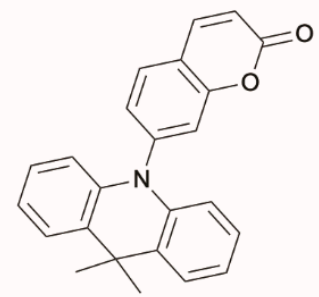

106

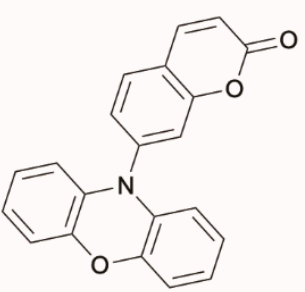

107

Scheme 36. Chemical structure of compounds 105-107.
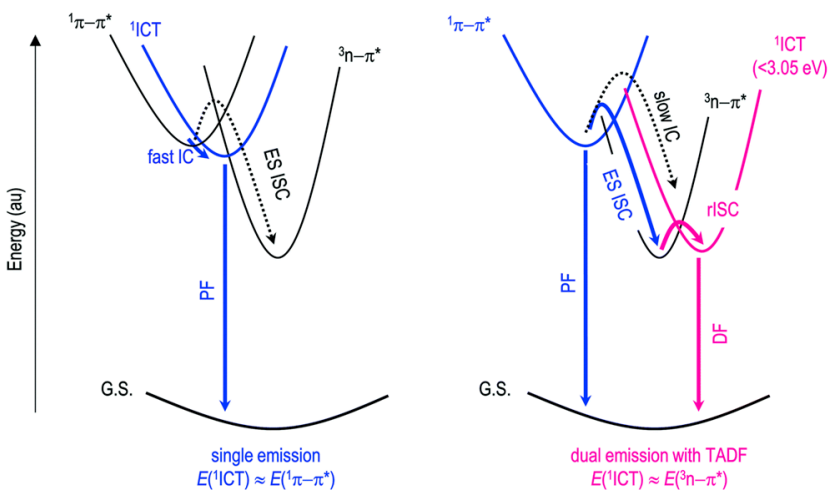

Figure 29. Mechanism proposed for the single Kasha D-coumarin derivatives (left) and dual antiKasha emission (right) from 105, 106 and 107. Abbreviations: G.S., ground state; ES ISC, El-Sayedrule-allowed ISC; PF, prompt fluorescence; DF, delayed fluorescence. Adapted from [87], Copyright 2021, with permission from the Royal Society of Chemistry.

The dual fluorescence was successfully used by the authors for ratiometric temperature monitoring in the 120-300 K range and triplet quencher detection.

Imran et al. [88] reported on the anti-Kasha behavior of radical cation 108 (Scheme 37). When excited at $537 \mathrm{~nm}$, DCM solutions of $\mathbf{1 0 8}$ display a red fluorescent emission centered at about $634 \mathrm{~nm}(\Phi=9.3 \%)$, which is intensified $(\Phi=19.3 \%)$ when the compound is dispersed in PMMA matrix (1 wt\%). The compound possesses one of the highest photo- 
stabilities among radical emitters with a half-life of $9.5 \times 10^{4} \mathrm{~s}$ under $350 \mathrm{~nm}$ irradiation. This property was ascribed to its cationic charge, which stabilizes ground and excited state molecular orbitals and inhibits their oxidation. DFT and configuration interaction singles calculations at the UB3LYP/6-31G+(d,p) level provided a low-energy excited doublet. According to the energy gap law, nonradiative deactivation was predicted from this state due to its closeness to the ground state. Therefore, the observed emission was associated with higher-energy calculated doublets, in violation of the Kasha rule.

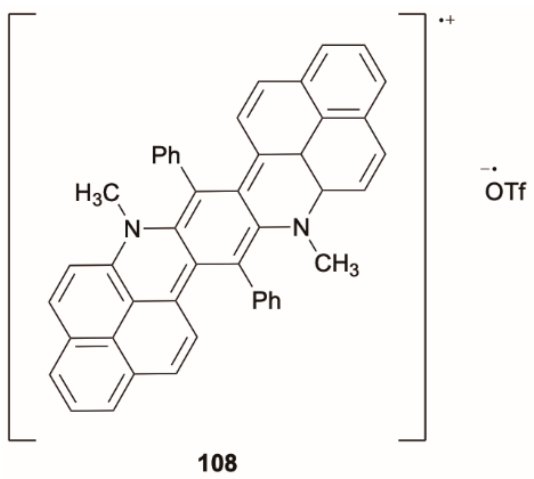

Scheme 37. Chemical structure of compound 108.

Multiple anti-Kasha emissions from three singlet states of a single molecule were reported by Franca et al. [89] for a spiro compound, 10-phenyl-10H,10 $H$-spiro[acridine9,9'-anthracen]-10'-one, 109 (Scheme 38), characterized by rigidly connected orthogonal D-A units. Excitation of toluene solutions of $\mathbf{1 0 9}$ at high energy provides simultaneous emission from three states (see Figure 30 ) which were described, based on previously reported calculations on this molecule [90], performed at the DFT/MRCI-R/SV(p) level, as $\mathrm{LE}_{\mathrm{D}}\left(\pi, \pi^{*}\right)\left({ }^{1} \mathrm{~B}_{1}\right.$ in Figure 30$), \mathrm{LE}_{\mathrm{A}}\left(n, \pi^{*}\right)$ and $\mathrm{CT}\left(\pi, \pi^{*}\right)$ states (in order of decreasing energy), the first two being locally excited states pertaining to the acridine (D) and the anthracenone (A) moieties, respectively. The lower-energy CT state, very close in energy to $\mathrm{LE}_{\mathrm{A}}$, provides both prompt and delayed fluorescence. The strongest allowed transition was ascribed to the high energy state, $\mathrm{LE}_{\mathrm{D}}$, located at about $0.6 \mathrm{eV}$ above the other two. Emission from $\mathrm{LE}_{\mathrm{D}}$ competes with both IC (to $\mathrm{LE}_{\mathrm{A}}$ and CT lower-lying states) and ISC to a close triplet state which then decays to populate a lower triplet state yielding DF from rISC. Direct population of the weaker states produces both prompt (from $\mathrm{LE}_{\mathrm{A}}$ and $\mathrm{CT}$ states) and delayed (from ISC between $\mathrm{LE}_{\mathrm{A}}$ and ${ }^{3} \mathrm{LE}_{\mathrm{A}}$ followed by rISC to CT state) emissions.

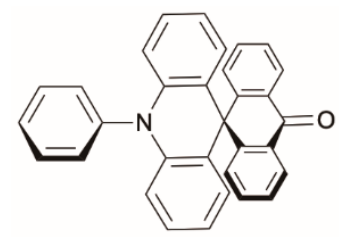

109

Scheme 38. Chemical structure of compound 109.

The existence of the three emissions was unequivocally assessed by working in aerated/deaerated conditions and by varying both the excitation wavelength, resulting in a change in the relative intensities of the emissions, and the solvent. Strong solvatochromic relaxation was observed for the lowest-energy singlet $C T$ state, while the close-lying $\mathrm{LE}_{\mathrm{A}}$ state was unaffected by polarity. The origin of such unprecedented rich photophysics of 109 was attributed to its rigid perpendicular geometry which effectively electronically decouples the $\mathrm{D}$ and $\mathrm{A}$ moieties. This ensures small $\Delta \mathrm{E}_{\mathrm{ST}}$ values and activates the TADF from a molecular CT state, in addition to the prompt emissions from the local $\mathrm{LE}_{\mathrm{D}}$ and $\mathrm{LE}_{\mathrm{A}}$ states. 


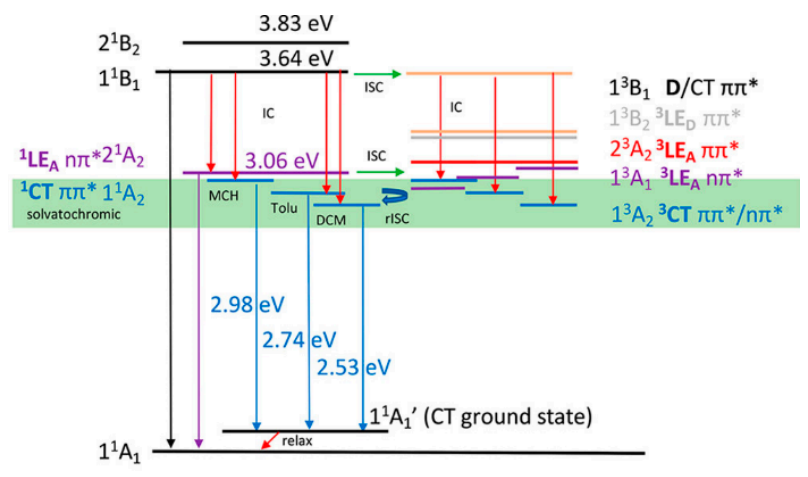

Figure 30. Measured energy levels from spectral onsets of $\mathbf{1 0 9}$ in solvents of different polarity. The green band represents the energy range over which solvatochromic states were observed. Red arrows represent nonradiative transitions. Adapted with permission from [89], Copyright 2021, American Chemical Society, https:/ / pubs.acs.org/doi/10.1021/acs.jpclett.0c03314 (accessed on 8 July 2021). Further permissions related to the material excerpted should be directed to the American Chemical Society.

\section{Conclusions}

This review of the literature of the past 10 years collects examples of purely organic compounds exhibiting photophysical behaviors that violate the Kasha rule. Mechanisms involved in different systems are frequently related to the same molecular properties, which are a large $E_{n}-E_{n-1}$ energy gap, a strong $E_{n}-E_{0}$ oscillator strength and a weak $E_{n-1^{-}}$ $\mathrm{E}_{0}$ oscillator strength $(n>1)$, where $\mathrm{E}$ refers to both singlet and triplet levels. Moreover, symmetry considerations on $E_{n}$ and $E_{n-1}$ have to be taken into account, together with the energy gap ones, as a factor that inhibits $E_{n}-E_{n-1} I C$, making the radiative deactivation from $E_{n}$ competitive. From the collected examples it appears that even a $\Delta E\left(E_{n}-E_{n-1}\right)$ as small as $0.35 \mathrm{eV}$ is enough to favor emission from $E_{n}$. This value is derived from computational studies, some of which can be of course further improved. However, at this stage, a $\Delta E\left(E_{n}-E_{n-1}\right)$ smaller than the generally accepted $1 \mathrm{eV}$ limit could be enough to observe anti-Kasha emission.

Alternatively, thermally activated anti-Kasha behavior may occur in the presence of a small $E_{n}-E_{n-1}$ energy gap (about $0.2 \mathrm{eV}$ ) by both reverse IC and reverse ISC.

In addition to basic research considerations, anti-Kasha behavior might offer improved performances useful for many practical applications. Among them, dual fluorescence from $S_{2}$ and $S_{1}$ and/or phosphorescence from $T_{2}$ and $T_{1}$ allow the development of OLEDs with dual electroluminescence based on a single compound, strongly simplifying device technology and concomitantly reducing fabrication costs. Other interesting issues are the intrinsic response of dual emitters to external stimuli such as temperature, allowing their use as fluorescence thermometers, or water, being optimal as humidity sensors, or encryption/decryption. The different lifetimes of their emitting states offer unprecedented opportunities for their use in time-resolved bio-imaging, solving the problem of detection in weak emission areas.

Author Contributions: Writing—review and editing: D.M., E.L., C.G., A.F., C.B. and E.C. All authors have read and agreed to the published version of the manuscript.

Funding: This research received no external funding.

Institutional Review Board Statement: Not applicable.

Informed Consent Statement: Not applicable.

Conflicts of Interest: The authors declare no conflict of interest. 


\begin{abstract}
Abbreviations
IC = internal conversion; ISC = intersystem crossing; RISC or rISC = reverse intersystem crossing; FRET = fluorescence resonance energy transfer; DFT = density functional theory; TDDFT = time-dependent density functional theory; CASPT2 = complete active space second-order perturbation theory; $\mathrm{MRCI}=$ multireference configuration interaction; ICT $=$ intermolecular charge transfer; $\mathrm{CT}=$ charge transfer; $\mathrm{AIE}=$ aggregation-induced emission; $\mathrm{CIE}=$ crystallization-induced emission; $\mathrm{QY}=$ quantum yield; $\mathrm{PMMA}$ = polymethylmetacrilate; $\mathrm{RT}=$ room temperature; $\mathrm{RTP}=$ room temperature phosphorescence; $\mathrm{DCM}=$ dichloromethane; $\mathrm{TA}=$ transient absorption; $\mathrm{EL}=$ electroluminescence; $\mathrm{DF}=$ delayed fluorescence; TADF = thermally activated delayed fluorescence; TAPC = 1,1'-bis(di-4-tolylaminophenyl)cyclohexane; TmPyPB = 1,3,5-tri(m-pyrid-3-yl-phenyl)benzene.
\end{abstract}

\title{
References
}

1. Kasha, M. Characterization of electronic transitions in complex molecules. Discuss. Faraday Soc. 1950, 9, 14-19. [CrossRef]

2. Beer, M.; Longuet-Higgins, H.C. Anomalous light emission of azulene. J. Chem. Phys. 1955, 23, 1390-1391. [CrossRef]

3. Klessinger, M.; Michl, J. Excited States and Photo-Chemistry of Organic Molecules; Revised and Improved English-Language Edition; Wiley-VCH Verlag GmbH \& Co. KGaA: New York, NY, USA, 1995; ISBN 978-0-471-18576-5.

4. Michl, J.; Thulstrup, E.W. Why is azulene blue and anthracene white? A simple mo picture. Tetrahedron 1976, 32, 205-209. [CrossRef]

5. Murata, S.; Iwanaga, C.; Toda, T.; Kokubun, H. Fluorescence yields of azulene derivatives. Chem. Phys. Lett. 1972, 13, 101-104. [CrossRef]

6. Maciejewski, A.; Steer, R.P. The Photophysics, Physical Photochemistry, and Related Spectroscopy of Thiocarbonyls. Chem. Rev. 1993, 93, 67-98. [CrossRef]

7. Aloisi, G.G.; Latterini, L.; Maçanita, A.L.; Becker, R.S.; Elisei, F. Singlet and triplet state properties of substituted flavothiones. Phys. Chem. Chem. Phys. 2003, 5, 3464-3469. [CrossRef]

8. Evans, R.C.; Douglas, P.; Winscom, C.J. Photophysics and electrochemistry of some thione far-red/near-IR triplet emitters. J. Fluoresc. 2009, 19, 169-177. [CrossRef]

9. Itoh, T. Fluorescence and Phosphorescence from Higher Excited States of Organic Molecules. Chem. Rev. 2012, 112, 4541-4568. [CrossRef]

10. Kondratenko, P.O.; Lopatkin, Y.M. On Anomalous Fluorescence of Symmetrical Polymethine Dyes. Int. J. Opt. 2020, $2020,6953206$. [CrossRef]

11. Behera, S.K.; Park, S.Y.; Gierschner, J. Dual Emission: Classes, Mechanisms, and Conditions. Angew. Chem. Int. Ed. 2021, 60, 2-17. [CrossRef] [PubMed]

12. Tseng, H.W.; Shen, J.Y.; Kuo, T.Y.; Tu, T.S.; Chen, Y.A.; Demchenko, A.P.; Chou, P.T. Excited-state intramolecular proton-transfer reaction demonstrating anti-Kasha behavior. Chem. Sci. 2016, 7, 655-665. [CrossRef]

13. Shafikov, M.Z.; Brandl, F.; Dick, B.; Czerwieniec, R. Can coumarins break kasha's rule? J. Phys. Chem. Lett. 2019, 10, 6468-6471. [CrossRef]

14. Del Valle, J.C.; Catalán, J. Kasha's rule: A reappraisal. Phys. Chem. Chem. Phys. 2019, 21, 10061-10069. [CrossRef] [PubMed]

15. Vavilov, S.I. XXIX. The dependence of the intensity of the fluorescence of dyes upon the wave-length of the exciting light. Lond. Edinb. Dublin Philos. Mag. J. Sci. 1922, 43, 307-320. [CrossRef]

16. Tozer, D.J.; Amos, R.D.; Handy, N.C.; Roos, B.O.; Serrano-Andres, L. Does density functional theory contribute to the understanding of excited states of unsaturated organic compounds? Mol. Phys. 1999, 97, 859-868. [CrossRef]

17. Fabian, J. Electronic excitation of sulfur-organic compounds-Performance of time-dependent density functional theory. Theor. Chem. Acc. 2001, 106, 199-217. [CrossRef]

18. Liao, M.S.; Lu, Y.; Parker, V.D.; Scheiner, S. DFT calculations and spectral measurements of charge-transfer complexes formed by aromatic amines and nitrogen heterocycles with tetracyanoethylene and chloranil. J. Phys. Chem. A 2003, 107, 8939-8948. [CrossRef]

19. Liao, M.S.; Lu, Y.; Scheiner, S. Performance assessment of density-functional methods for study of charge-transfer complexes. J. Comput. Chem. 2003, 24, 623-631. [CrossRef]

20. Tozer, D.J. Relationship between long-range charge-transfer excitation energy error and integer discontinuity in Kohn-Sham theory. J. Chem. Phys. 2003, 119, 12697-12699. [CrossRef]

21. Dreuw, A.; Head-Gordon, M. Failure of Time-Dependent Density Functional Theory for Long-Range Charge-Transfer Excited States: The Zincbacteriochlorin-Bacteriochlorin and Bacteriochlorophyll-Spheroidene Complexes. J. Am. Chem. Soc. 2004, 126, 4007-4016. [CrossRef] [PubMed]

22. Johnson, L.E.; Dalton, L.R.; Robinson, B.H. Optimizing Calculations of Electronic Excitations and Relative Hyperpolarizabilities of Electrooptic Chromophores. Acc. Chem. Res. 2014, 47, 3258-3265. [CrossRef]

23. Kümmel, S.; Kronik, L. Orbital-dependent density functionals: Theory and applications. Rev. Mod. Phys. 2008, 80, 3-60. [CrossRef] 
24. Leininger, T.; Stoll, H.; Werner, H.J.; Savin, A. Combining long-range configuration interaction with short-range density functional. Chem. Phys. Lett. 1997, 275, 151-160. [CrossRef]

25. Yanai, T.; Tew, D.P.; Handy, N.C. A new hybrid exchange-correlation functional using the Coulomb-attenuating method (CAMB3LYP). Chem. Phys. Lett. 2004, 393, 51-57. [CrossRef]

26. Iikura, H.; Tsuneda, T.; Yanai, T.; Hirao, K. A long-range correction scheme for generalized-gradient-approximation exchange functionals. J. Chem. Phys. 2001, 115, 3540-3544. [CrossRef]

27. Baer, R.; Neuhauser, D. Density functional theory with correct long-range asymptotic behavior. Phys. Rev. Lett. 2005, 94, 043002. [CrossRef]

28. Stein, T.; Kronik, L.; Baer, R. Reliable prediction of charge transfer excitations in molecular complexesusing time-dependent density functional theory. J. Am. Chem. Soc. 2009, 131, 2818-2820. [CrossRef]

29. Kümmel, S. Charge-Transfer Excitations: A Challenge for Time-Dependent Density Functional Theory That Has Been Met. Adv. Energy Mater. 2017, 7, 1700440. [CrossRef]

30. Maitra, N.T. Charge transfer in time-dependent density functional theory. J. Phys. Condens. Matter 2017, 29, 423001. [CrossRef]

31. Jacquemin, D.; Planchat, A.; Adamo, C.; Mennucci, B. TD-DFT assessment of functionals for optical 0-0 transitions in solvated dyes. J. Chem. Theory Comput. 2012, 8, 2359-2372. [CrossRef] [PubMed]

32. Steer, R.P. Photophysics of molecules containing multiples of the azulene carbon framework. J. Photochem. Photobiol. C Photochem. Rev. 2019, 40, 68-80. [CrossRef]

33. Xin, H.; Hou, B.; Gao, X. Azulene-Based $\pi$-Functional Materials: Design, Synthesis, and Applications. Acc. Chem. Res. 2021, 54, 1737-1753. [CrossRef] [PubMed]

34. Veys, K.; Escudero, D. Computational Protocol to Predict Anti-Kasha Emissions: The Case of Azulene Derivatives. J. Phys. Chem. A 2020, 124, 7228-7237. [CrossRef]

35. Prlj, A.; Begušić, T.; Zhang, Z.T.; Fish, G.C.; Wehrle, M.; Zimmermann, T.; Choi, S.; Roulet, J.; Moser, J.E.; Vaníček, J. Semiclassical Approach to Photophysics beyond Kasha's Rule and Vibronic Spectroscopy beyond the Condon Approximation. The Case of Azulene. J. Chem. Theory Comput. 2020, 16, 2617-2626. [CrossRef] [PubMed]

36. Xin, H.; Gao, X. Application of Azulene in Constructing Organic Optoelectronic Materials: New Tricks for an Old Dog. Chempluschem 2017, 82, 945-956. [CrossRef]

37. Zhou, Y.; Zhuang, Y.; Li, X.; Ågren, H.; Yu, L.; Ding, J.; Zhu, L. Selective Dual-Channel Imaging on Cyanostyryl-Modified Azulene Systems with Unimolecularly Tunable Visible-Near Infrared Luminescence. Chem. Eur. J. 2017, 23, 7642-7647. [CrossRef]

38. Gong, Y.; Zhou, Y.; Yue, B.; Wu, B.; Sun, R.; Qu, S.; Zhu, L. Multiwavelength anti-kasha's rule emission on self-assembly of azulene-functionalized persulfurated arene. J. Phys. Chem. C 2019, 123, 22511-22518. [CrossRef]

39. Zhou, Y.; Baryshnikov, G.; Li, X.; Zhu, M.; Ågren, H.; Zhu, L. Anti-Kasha's Rule Emissive Switching Induced by Intermolecular H-Bonding. Chem. Mater. 2018, 30, 8008-8016. [CrossRef]

40. Zhang, X.S.; Huang, Y.Y.; Zhang, J.; Meng, W.; Peng, Q.; Kong, R.; Xiao, Z.; Liu, J.; Huang, M.; Yi, Y.; et al. Dicyclohepta [ijkl, uvwx] Rubicene with Two Pentagons and Two Heptagons as a Stable and Planar Non-Benzenoid Nanographene. Angew. Chem. Int. Ed. 2020, 59, 3529-3533. [CrossRef]

41. Amir, E.; Amir, R.J.; Campos, L.M.; Hawker, C.J. Stimuli-responsive azulene-based conjugated oligomers with polyaniline-like properties. J. Am. Chem. Soc. 2011, 133, 10046-10049. [CrossRef] [PubMed]

42. Koch, M.; Blacque, O.; Venkatesan, K. Syntheses and Tunable Emission Properties of 2-Alkynyl Azulenes. Org. Lett. 2012, 14, 1580-1583. [CrossRef] [PubMed]

43. Shoji, T.; Araki, T.; Iida, N.; Miura, K.; Ohta, A.; Sekiguchi, R.; Ito, S.; Okujima, T. Synthesis of azulenophthalimides by phosphine-mediated annulation of 1,2-diformylazulenes with maleimides. Org. Chem. Front. 2019, 6, 195-204. [CrossRef]

44. Shoji, T.; Sugiyama, S.; Kobayashi, Y.; Yamazaki, A.; Ariga, Y.; Katoh, R.; Wakui, H.; Yasunami, M.; Ito, S. Direct synthesis of 2-arylazulenes by [8+2] cycloaddition of $2 \mathrm{H}$-cyclohepta [b] furan-2-ones with silyl enol ethers. Chem. Commun. 2020, 56, 1485-1488. [CrossRef] [PubMed]

45. Xin, H.; Li, J.; Yang, X.; Gao, X. Azulene-Based BN-Heteroaromatics. J. Org. Chem. 2020, 85, 70-78. [CrossRef]

46. Xin, H.; Li, J.; Lu, R.Q.; Gao, X.; Swager, T.M. Azulene-Pyridine-Fused Heteroaromatics. J. Am. Chem. Soc. 2020, 142, 13598-13605. [CrossRef]

47. Lucenti, E.; Forni, A.; Botta, C.; Carlucci, L.; Giannini, C.; Marinotto, D.; Previtali, A.; Righetto, S.; Cariati, E. H-Aggregates Granting Crystallization-Induced Emissive Behavior and Ultralong Phosphorescence from a Pure Organic Molecule. J. Phys. Chem. Lett. 2017, 8, 1894-1898. [CrossRef]

48. Lucenti, E.; Forni, A.; Botta, C.; Carlucci, L.; Giannini, C.; Marinotto, D.; Pavanello, A.; Previtali, A.; Righetto, S.; Cariati, E. Cyclic Triimidazole Derivatives: Intriguing Examples of Multiple Emissions and Ultralong Phosphorescence at Room Temperature. Angew. Chem. Int. Ed. 2017, 56, 16302-16307. [CrossRef]

49. Giannini, C.; Forni, A.; Malpicci, D.; Lucenti, E.; Marinotto, D.; Previtali, A.; Carlucci, L.; Cariati, E. Room Temperature Phosphorescence from Organic Materials: Unravelling the Emissive Behaviour of Chloro-Substituted Derivatives of Cyclic Triimidazole. Eur. J. Org. Chem. 2021, 2021, 2041-2049. [CrossRef]

50. Lucenti, E.; Forni, A.; Botta, C.; Carlucci, L.; Colombo, A.; Giannini, C.; Marinotto, D.; Previtali, A.; Righetto, S.; Cariati, E. The Effect of Bromo Substituents on the Multifaceted Emissive and Crystal-Packing Features of Cyclic Triimidazole Derivatives. ChemPhotoChem 2018, 2, 801-805. [CrossRef] 
51. Lucenti, E.; Forni, A.; Previtali, A.; Marinotto, D.; Malpicci, D.; Righetto, S.; Giannini, C.; Virgili, T.; Kabacinski, P.; Ganzer, L.; et al. Unravelling the intricate photophysical behavior of 3-(pyridin-2-yl) triimidazotriazine AIE and RTP polymorphs. Chem. Sci. 2020, 11, 7599-7608. [CrossRef]

52. Yang, S.; Cao, C.; Islam, A.; Sun, S.; Deng, Z.; Li, J.; Ni, S.; Tong, Q.X.; Li, M. De Disentangling Multiple Effects on ExcitedState Intramolecular Charge Transfer among Asymmetrical Tripartite PPI-TPA/PCz Triads. Chem. Eur. J. 2021, 27, $1337-1345$. [CrossRef] [PubMed]

53. Qiao, X.; Liu, Y.; Yao, J.; He, X.; Liu, H.; Lu, P.; Ma, D. Upper Excited Triplet State-Mediated Intersystem Crossing for Anti-Kasha's Fluorescence: Potential Application in Deep-Ultraviolet Sensing. J. Phys. Chem. C 2019, 123, 5761-5766. [CrossRef]

54. Jiang, Q.; Xu, Y.; Liang, X.; Wang, C.; Tang, X.; Li, Y.; Qiu, X.; Zheng, N.; Zhao, R.; Zhao, D.; et al. Stable High-Energy Excited States Observed in a Conjugated Molecule with Hindered Internal Conversion Processes. J. Phys. Chem. C 2019, 123, 6190-6196. [CrossRef]

55. Das, D.K.; Makhal, K.; Singhal, S.; Goswami, D. Polarization induced control of multiple fluorescence from a molecule. Chem. Phys. Lett. 2013, 579, 45-50. [CrossRef]

56. Das, D.K.; Makhal, K.; Goswami, D. Solvent effect on multiple emission and ultrafast dynamics of higher excited states. Chem. Phys. Lett. 2018, 706, 375-379. [CrossRef]

57. Nairat, M.; Konar, A.; Lozovoy, V.V.; Beck, W.F.; Blanchard, G.J.; Dantus, M. Controlling S2 Population in Cyanine Dyes Using Shaped Femtosecond Pulses. J. Phys. Chem. A 2016, 120, 1876-1885. [CrossRef]

58. Guarin, C.A.; Villabona-Monsalve, J.P.; López-Arteaga, R.; Peon, J. Dynamics of the higher lying excited states of cyanine dyes. An ultrafast fluorescence study. J. Phys. Chem. B 2013, 117, 7352-7362. [CrossRef]

59. Kumari, A.; Gupta, S. Two-photon excitation and direct emission from S2 state of U.S. Food and Drug Administration approved near-infrared dye: Application of anti-Kasha's rule for two-photon fluorescence imaging. J. Biophotonics 2019, 12, 1-7. [CrossRef]

60. Sadiq, F.; Zhao, J.; Hussain, M.; Wang, Z. Effect of thiophene substitution on the intersystem crossing of arene photosensitizers. Photochem. Photobiol. Sci. 2018, 17, 1794-1803. [CrossRef]

61. Chaudhuri, D.; Sigmund, E.; Meyer, A.; Röck, L.; Klemm, P.; Lautenschlager, S.; Schmid, A.; Yost, S.R.; Vanvoorhis, T.; Bange, S.; et al. Metal-free OLED triplet emitters by side-stepping Kasha's rule. Angew. Chem. Int. Ed. 2013, 52, 13449-13452. [CrossRef]

62. He, Z.; Zhao, W.; Lam, J.W.Y.; Peng, Q.; Ma, H.; Liang, G.; Shuai, Z.; Tang, B.Z. White light emission from a single organic molecule with dual phosphorescence at room temperature. Nat. Commun. 2017, 8, 1-7. [CrossRef] [PubMed]

63. Paul, L.; Moitra, T.; Ruud, K.; Chakrabarti, S. Strong Duschinsky Mixing Induced Breakdown of Kasha's Rule in an Organic Phosphor. J. Phys. Chem. Lett. 2019, 10, 369-374. [CrossRef] [PubMed]

64. Kupka, H.; Cribb, P.H. Multidimensional Franck-Condon integrals and Duschinsky mixing effects. J. Chem. Phys. 1986, 85, 1303-1315. [CrossRef]

65. Cao, J.; Li, S.; Wang, H.C.; Bai, S.J.; Wang, Z.; Ren, X.; Xu, Y.X. Distinct luminescent properties between thiophene-S-oxide and Thiophene-S, S-dioxides incorporated ladder-type molecules. Dyes Pigments 2020, 175, 108147. [CrossRef]

66. Wex, B.; Kaafarani, B.R. Perspective on carbazole-based organic compounds as emitters and hosts in TADF applications. J. Mater. Chem. C 2017, 5, 8622-8653. [CrossRef]

67. Feng, C.; Fu, H.; Yao, J.; Wu, Y.; Li, S.; Fu, L.; Xiao, X.; Xu, Z.; Liao, Q. Breaking kasha's rule as a mechanism for solution-phase room-temperature phosphorescence from high-lying triplet excited state. J. Phys. Chem. Lett. 2020, 11, 8246-8251. [CrossRef]

68. Wang, T.; Su, X.; Zhang, X.; Nie, X.; Huang, L.; Zhang, X.; Sun, X.; Luo, Y.; Zhang, G. Aggregation-Induced Dual-Phosphorescence from Organic Molecules for Nondoped Light-Emitting Diodes. Adv. Mater. 2019, 31, 1-7. [CrossRef]

69. Wilson, M.W.B.; Rao, A.; Clark, J.; Kumar, R.S.S.; Brida, D.; Cerullo, G.; Friend, R.H. Ultrafast dynamics of exciton fission in polycrystalline pentacene. J. Am. Chem. Soc. 2011, 133, 11830-11833. [CrossRef]

70. Itoh, T. Phosphorescence from the T2 $\left(n, \pi^{*}\right)$ state observed for 4-hyroxybenzaldehyde in a p-dichlorobenzene matrix. Chem. Phys. Lett. 2014, 591, 109-112. [CrossRef]

71. García, R.; More, S.; Melle-Franco, M.; Mateo-Alonso, A. 11,11,12,12-tetracyano-4,5-pyrenoquinodimethanes: Air-stable push-pull o-quinodimethanes with S2 fluorescence. Org. Lett. 2014, 16, 6096-6099. [CrossRef]

72. Arimori, S.; Davidson, M.G.; Fyles, T.M.; Hibbert, T.G.; James, T.D.; Kociok-Köhn, G.I. Synthesis and structural characterisation of the first bis(bora)calixarene: A selective, bidentate, fluorescent fluoride sensor. Chem. Commun. 2004, 4, 1640-1641. [CrossRef] [PubMed]

73. Jin, J.; Park, J.Y.; Lee, Y.S. Optical Nature and Binding Energetics of Fluorescent Fluoride Sensor Bis(bora)calix[4]arene and Design Strategies of Its Homologues. J. Phys. Chem. C 2016, 120, 24324-24334. [CrossRef]

74. Qian, H.; Cousins, M.E.; Horak, E.H.; Wakefield, A.; Liptak, M.D.; Aprahamian, I. Suppression of Kasha's rule as a mechanism for fluorescent molecular rotors and aggregation-induced emission. Nat. Chem. 2017, 9, 83-87. [CrossRef] [PubMed]

75. Zhou, P.; Li, P.; Zhao, Y.; Han, K. Restriction of Flip-flop Motion as a Mechanism for Aggregation-Induced Emission. J. Phys. Chem. Lett. 2019, 10, 6929-6935. [CrossRef] [PubMed]

76. Zhou, C.; Zhang, S.; Gao, Y.; Liu, H.; Shan, T.; Liang, X.; Yang, B.; Ma, Y. Ternary Emission of Fluorescence and Dual Phosphorescence at Room Temperature: A Single-Molecule White Light Emitter Based on Pure Organic Aza-Aromatic Material. Adv. Funct. Mater. 2018, 28, 1-6. [CrossRef]

77. Chen, J.; Chen, Y.; Wu, Y.; Wang, X.; Yu, Z.; Xiao, L.; Liu, Y.; Tian, H.; Yao, J.; Fu, H. Modulated emission from dark triplet excitons in aza-acene compounds: Fluorescence versus phosphorescence. New J. Chem. 2017, 41, 1864-1871. [CrossRef] 
78. Peng, Z.; Wang, Z.; Huang, Z.; Liu, S.; Lu, P.; Wang, Y. Expression of anti-Kasha's emission from amino benzothiadiazole and its utilization for fluorescent chemosensors and organic light emitting materials. J. Mater. Chem. C 2018, 6, 7864-7873. [CrossRef]

79. Sun, Z.B.; Liu, J.K.; Yuan, D.F.; Zhao, Z.H.; Zhu, X.Z.; Liu, D.H.; Peng, Q.; Zhao, C.H. 2,2'-Diamino-6,6'-Diboryl-1,1'-Binaphthyl: A Versatile Building Block for Temperature-Dependent Dual Fluorescence and Switchable Circularly Polarized Luminescence. Angew. Chem. Int. Ed. 2019, 58, 4840-4846. [CrossRef]

80. Liu, D.H.; Sun, Z.B.; Zhao, Z.H.; Peng, Q.; Zhao, C.H. 1,1'-Binaphthyl Consisting of Two Donor- $\pi$-Acceptor Subunits: A General Skeleton for Temperature-Dependent Dual Fluorescence. Chem. Eur. J. 2019, 25, 10179-10187. [CrossRef]

81. Liu, Z.Y.; Hu, J.W.; Huang, C.H.; Huang, T.H.; Chen, D.G.; Ho, S.Y.; Chen, K.Y.; Li, E.Y.; Chou, P.T. Sulfur-Based Intramolecular Hydrogen-Bond: Excited-State Hydrogen-Bond On/Off Switch with Dual Room-Temperature Phosphorescence. J. Am. Chem. Soc. 2019, 141, 9885-9894. [CrossRef]

82. Guo, J.; Fan, J.; Lin, L.; Zeng, J.; Liu, H.; Wang, C.K.; Zhao, Z.; Tang, B.Z. Mechanical Insights into Aggregation-Induced Delayed Fluorescence Materials with Anti-Kasha Behavior. Adv. Sci. 2019, 6, 1801629. [CrossRef]

83. Shi, L.; Yan, C.; Guo, Z.; Chi, W.; Wei, J.; Liu, W.; Liu, X.; Tian, H.; Zhu, W.H. De novo strategy with engineering anti-Kasha/Kasha fluorophores enables reliable ratiometric quantification of biomolecules. Nat. Commun. 2020, 11, 1-11. [CrossRef]

84. Luo, M.; Li, X.; Ding, L.; Baryshnikov, G.; Shen, S.; Zhu, M.; Zhou, L.; Zhang, M.; Lu, J.; Ågren, H.; et al. Integrating Time-Resolved Imaging Information by Single-Luminophore Dual Thermally Activated Delayed Fluorescence. Angew. Chem. Int. Ed. 2020, 59, 17018-17025. [CrossRef]

85. Wu, Y.H.; Xiao, H.; Chen, B.; Weiss, R.G.; Chen, Y.Z.; Tung, C.H.; Wu, L.Z. Multiple-State Emissions from Neat, Single-Component Molecular Solids: Suppression of Kasha's Rule. Angew. Chem. Int. Ed. 2020, 59, 10173-10178. [CrossRef] [PubMed]

86. Sun, H.; Sun, S.S.; Han, F.F.; Zhao, Y.; Li, M.D.; Miao, B.X.; Nie, J.; Zhang, R.; Ni, Z.H. Water-stimuli-responsive dynamic fluorescent switch from Kasha's rule to anti-Kasha's rule based on a tetraphenylethene substituted Schiff base. Chem. Eng. J. 2021, 405, 127000. [CrossRef]

87. Jhun, B.H.; Jeong, D.Y.; Nah, S.; Park, S.Y.; You, Y. Novel anti-Kasha fluorophores exhibiting dual emission with thermally activated delayed fluorescence through detouring triplet manifolds. J. Mater. Chem. C 2021, 9, 7083-7093. [CrossRef]

88. Imran, M.; Wehrmann, C.M.; Chen, M.S. Open-Shell Effects on Optoelectronic Properties: Antiambipolar Charge Transport and Anti-Kasha Doublet Emission from a N-Substituted Bisphenalenyl. J. Am. Chem. Soc. 2020, 142, 38-43. [CrossRef]

89. Franca, L.G.; Long, Y.; Li, C.; Danos, A.; Monkman, A. The Critical Role of $\mathrm{n} \pi *$ States in the Photophysics and Thermally Activated Delayed Fluorescence of Spiro Acridine-Anthracenone. J. Phys. Chem. Lett. 2021, 12, 1490-1500. [CrossRef]

90. Lyskov, I.; Marian, C.M. Climbing up the ladder: Intermediate triplet states promote the reverse intersystem crossing in the efficient TADF emitter ACRSA. J. Phys. Chem. C 2017, 121, 21145-21153. [CrossRef] 